\title{
Thiopurines' Metabolites and Drug Toxicity: A Meta-Analysis
}

\author{
Paula Sousa ${ }^{1}$, Maria Manuela Estevinho ${ }^{2,3}$, Cláudia Camila Dias ${ }^{4,5}$, Paula Ministro ${ }^{1}$, \\ Uri Kopylov ${ }^{6}$, Silvio Danese ${ }^{7,8}$, Laurent Peyrin-Biroulet ${ }^{9}$ and Fernando Magro ${ }^{2,10,11, *(\mathbb{C})}$ \\ 1 Department of Gastroenterology, Viseu Unit, Tondela-Viseu Hospital Centre, 3504-509 Viseu, Portugal; \\ paula.sousa.6377@hstviseu.min-saude.pt (P.S.); paula.ministro.4342@hstviseu.min-saude.pt (P.M.) \\ 2 Department of Biomedicine, Unit of Pharmacology and Therapeutics, University of Porto, \\ 4200-450 Porto, Portugal; mmestevinho@gmail.com \\ 3 Department of Gastroenterology, Centro Hospitalar Vila Nova de Gaia/Espinho, \\ 4434-502 Vila Nova de Gaia, Portugal \\ 4 Department of Community Medicine, Information and Decision in Health, University of Porto, \\ 4200-450 Porto, Portugal; ccamiladias@gmail.com \\ 5 Centre for Health Technology and Services Research, University of Porto, 4200-450 Porto, Portugal \\ 6 Department of Gastroenterology, Sheba Medical Central, Ramat Gan and Sackler Medical School, \\ Tel Aviv University, 52621 Ramat Gan, Israel; ukopylov@gmail.com \\ 7 Department of Biomedical Sciences, Humanitas University, 20090 Milan, Italy; silvio.danese@hunimed.eu \\ 8 Humanitas Clinical and Research Center, IRCCS, 20089 Milan, Italy \\ 9 Department of Gastroenterology, Nancy University Hospital, University of Lorraine, \\ 54500 Vandoeuvre-lès-Nancy, France; peyrinbiroulet@gmail.com \\ 10 Department of Gastroenterology, São João University Hospital, 4200-319 Porto, Portugal \\ 11 MedInUP, Centre for Drug Discovery and Innovative Medicines, 4200-319 Porto, Portugal \\ * Correspondence: fm@med.up.pt; Tel.: +351-22-552-3600
}

Received: 24 May 2020; Accepted: 9 July 2020; Published: 13 July 2020

check for updates

\begin{abstract}
Many questions remain unanswered regarding therapeutic drug monitoring (TDM) utility with thiopurines. This study aims to establish a relationship between thiopurines' metabolites and drug toxicity. We performed a systematic review with inclusion of studies evaluating the relationship between thiopurines' metabolites and drug toxicity. Meta-analysis of mean difference (MD), correlations and odds ratio (OR) was performed. We identified 21,240 records, 72 of which were eligible for meta-analysis. Levels of 6-thioguanine nucleotides (6-TGN) were higher in patients with leukopenia (MD $127.06 \mathrm{pmol} / 8 \times 10^{8} \mathrm{RBC}$ ) and gastrointestinal intolerance (MD $201.46 \mathrm{pmol} / 8$ $\times 10^{8} \mathrm{RBC}$ ), and lower in patients with hepatotoxicity (MD $\left.-40.6 \mathrm{pmol} \times 10^{8} \mathrm{RBC}\right)$. We established a significant correlation between 6-TGN and leukocytes $(r=-0.21)$, neutrophils $(r=-0.24)$ and alanine aminotransferase levels $(\mathrm{r}=-0.24)$. OR for leukopenia in patients with elevated 6-TGN was 4.63 (95\% CI 2.24; 9.57). An optimal cut-off of $135 \mathrm{pmol} / 8 \times 10^{8} \mathrm{RBC}$ for leukopenia was calculated (sensitivity 75.4\%; specificity 46.4\%). 6-methylmercaptopurine ribonucleotides (6-MMPR) were significantly associated with hepatotoxicity (MD $3241.2 \mathrm{pmol} / 8 \times 10^{8} \mathrm{RBC}$; OR 4.28; 95\% CI 3.20; 5.71). Levels of 6-MMPR measured in the first 8 weeks of treatment were associated with leukopenia. We conclude that TDM could be used to prevent thiopurines' toxicity. As optimal metabolites level may vary according to indication, physicians may adapt posology to decrease toxicity without compromising efficacy.
\end{abstract}

Keywords: thiopurines; therapeutic drug monitoring; adverse events 


\section{Introduction}

Thiopurines (comprising azathioprine (AZA), 6-mercaptopurine (6-MP), and 6-thioguanine) have been used for over 5 decades in the treatment of a myriad of disorders, including acute lymphoblastic leukemia (ALL), inflammatory bowel disease (IBD), auto-immune hepatitis (AIH), and also in the prophylaxis of rejection in organ transplant recipients [1].

As prodrugs, thiopurines have a complex metabolism which leads to the formation of 6-thioguanine nucleotides (6-TGN). Regarding conventional thiopurines, other pathways compete with the production of the active metabolite 6-TGN, leading to the formation of 6-methylmercaptopurine (6-MMP) and 6-MMP ribonucleotides (6-MMPR). These metabolites can be determined by different methods, such as the Lennard [2] and Dervieux-Boulieu assays [3], that perform the measurement in red blood cells (RBC), with concentrations expressed as $\mathrm{pmol} / 8 \times 10^{8} \mathrm{RBC}$.

Thiopurines present toxicity at distinct levels: myelosuppression, hepatotoxicity, pancreatitis and gastrointestinal intolerance, among others. Toxicity is an important cause of treatment cessation; in IBD, about $15 \%$ of patients discontinue thiopurines due to adverse events [4,5]. The toxicity of thiopurines can be divided into dose-dependent and idiosyncratic. Due to the distinct metabolisms, the safety profiles of thiopurines may differ. The most worrisome adverse event of 6-thioguanine is liver nodular regenerative hyperplasia (NRH), which still detracts some physicians from its use [6].

The balance between efficacy and toxicity can be achieved with tailored dosing and monitoring, using a weight-based regimen. However, the dose of thiopurines does not correlate with the levels of metabolites [7]. The level of metabolites, specifically 6-TGN, has been associated with improved clinical outcomes in ALL, renal transplantation, and IBD [8-11]. An optimal therapeutic range of $\sim 230$ to $400 \mathrm{pmol} / 8 \times 10^{8} \mathrm{RBC}$ is often cited for patients with IBD and other disorders [12,13]. Values of 6-TGN of $450 \mathrm{pmol} / 8 \times 10^{8} \mathrm{RBC}$ and of 6-MMPR of $5700 \mathrm{pmol} / 8 \times 10^{8} \mathrm{RBC}$ were reported as thresholds for myelotoxicity and hepatotoxicity, respectively $[14,15]$. However, the benefit of therapeutic drug monitoring (TDM) for thiopurines is still uncertain [16]. Based on the risk of myelosuppression, Food and Drug Administration (FDA) and the Clinical Pharmacogenetics Implementation Consortium recommend genotyping or phenotyping for thiopurine S-methyltransferase (TPMT) deficiency prior to starting thiopurines [17-19]. American Gastroenterology Association and proceedings of the first Thiopurine Task Force meeting $[6,16]$ state that the benefit for routine TPMT testing is still uncertain for most patients, and some real-life studies support this statement [20].

Even though this topic is of great interest and can influence the clinical practice in several disciplines, as far as we know, scientific literature lacks a comprehensive study on the metabolites of thiopurines and their correlation with toxicity. The aim of this study was to conduct a systematic review and meta-analysis of the studies associating the levels of thiopurines' metabolites with the occurrence of toxicity.

\section{Materials and Methods}

\subsection{Search Strategy}

In this study, we followed the Preferred Reporting Items for Systematic Reviews and Meta-Analyses (PRISMA) [21] and the Cochrane Collaboration [22] Guidelines. On November 2018, we performed a literature search on three electronic databases: Pubmed [23], Web of Science [24] and Scopus [25], without time restrictions. The following search words or Medical Subject Heading terms were used: (("Azathioprine" OR "Mercaptopurine" OR "6-thioguanine nucleotide" OR "6-methyl mercaptopurine") AND ("Drug-Related Side Effects and Adverse Reactions" OR "adverse effects" OR "leukopenia" OR "toxicity" OR "infection" OR "hepatotoxicity")). We performed a manual search of the list of references of all relevant studies to ensure that all pertinent articles were considered. 


\subsection{Eligibility and Inclusion/Exclusion Criteria}

For our meta-analysis, we considered eligible for inclusion all the studies enrolling adult and/or pediatric patients in which the relationship between thiopurines' metabolites and toxicity was evaluated. The underlying disease was not a factor for eligibility. The following types of toxicity were considered: myelotoxicity (subdivided in leukopenia, lymphopenia, neutropenia, anemia, and thrombocytopenia), hepatotoxicity, infections, pancreatitis, and/or gastrointestinal intolerance. Oncological adverse events, effects in pregnancy or offspring outcomes, and postsurgical complications were not considered. Randomized controlled trials, cohort studies and case series with more than five patients were considered. No restriction in terms of publication dates was applied. Concomitant medications were allowed but listed.

The exclusion criteria were: (i) systematic reviews or guidelines; (ii) animal studies; (iii) individual case reports; and (iv) case series with up to five patients.

\subsection{Study Selection and Data Collection}

First, we screened the list of titles and the abstracts of the studies identified in the initial search. The list of references was screened by two reviewers and all studies not fulfilling the inclusion criteria were excluded from further analysis. In cases of disagreement, a third independent reviewer was consulted. For the remaining studies, we analyzed full text articles to determine eligibility. Rayyan application (Qatar Computing Research Institute, Doha, Qatar) was used during this process [26].

From the selected studies, we collected the following information: journal and authors' name, publication year, type of study, cohort's geographic origin, cohort's age group (pediatric vs. adult), number of enrolled patients, number of patients with measurement of metabolites, underlying disease for which the thiopurine was used, type of thiopurine and treatment duration, concomitant medication, 6-TGN and 6-MMPR serum levels and cut-offs, methodology used for metabolites quantification, time of metabolites quantification, type of toxicity, definition of toxicity, relationship between thiopurines' metabolites and drug toxicity.

\subsection{Quality Assessment}

We used funnel plot analysis to detect potential publication bias and/or systematic heterogeneity [22]. The quality of the studies was assessed following the quality assessment tool (QATSDD, Sirriyeh et al, Leeds, UK) [27]. For each study, the scores were added and divided by the maximum possible score (42) to obtain the overall quality score.

\subsection{Statistical Analysis}

In this meta-analysis, the main variable was the occurrence of toxicity. Three types of data were available:

1. Mean values of metabolites concentration in patients with or without toxicity

Since many studies provided medians, in order to avoid losing a significant amount of data by excluding them from the analysis, mean and standard deviation (SD) were calculated from the reported data, as described by Wan et al. (2014) [28]. The studies in which this calculation was performed are identified with an asterisk in the forest-plots. Studies providing full data-allowing the direct calculation of mean and SD - but with a skewed distribution of the variables are identified with two asterisks in the forest-plots. When data were only presented in the form of an image, we extracted the values with WebPlotDigitizer v4.2 (Ankit Rohatgi, Pacifica, CA, USA). Random-effects models were used to test whether mean 6-TGN, 6-MMP or ratio 6-MMPR/TGN values differed among patients with and without toxicity. Review manager v5.3 (Cochrane, London, UK) was used for the evaluation of mean metabolites/ratio differences. 


\section{2. $\quad$ Odds Ratio (OR)}

When available, the proportion of patients with and without a specific toxicity with levels of 6-TGN/6-MMPR/ratio above and below the defined threshold values was extracted or calculated from each article. However, some studies only provided the final OR value. In these cases, when available, the OR from multivariate analysis was included. Stata 16 (StataCorp, Lakeway Drive, TX, USA) was used for estimating the pooled OR and its 95\% confidence interval (95\% CI), using a random-effects model. Since different studies used different methods to measure 6-TGN, a previously recommended strategy was used to compare data [29]. The Lennard assay was used as "standard" [2,30,31]; the values of 6-TGN in studies using the Dervieux-Boulieu assay [3] were divided by 2.6 [29], and those obtained with the Erdmann method [32] and with the commercial assay offered by Prometheus Laboratories Inc. (San Diego, CA, USA) were multiplied by 1.6 [33,34]. A high degree of correlation between methodologies has been demonstrated [31,33]. Concentrations of 6-MMPR were not converted, as it has been reported that these are similar in different assays [35-37].

\section{Correlations}

Since some toxicities can be presented as continuous variables, correlation analysis was also performed. The correlation coefficient was extracted or calculated from each article. The Schmidt-Hunter method was used to calculate the overall correlation coefficient ( $r$ ) from a set of correlations [38]. Statsdirect v3.2.8 (StatsDirect Ltd., Birkenhead, Merseyside, UK) was used for this analysis.

All the presented $p$-values are two-sided and have a 5\% significance level. Statistical heterogeneity was assessed using the $\mathrm{I}^{2}$ statistic and by performing subgroup analyses on the following variables: (i) methodology used for the determination of metabolites; (ii) age group; (iii) underlying disease; (iv) geographical origin of the studies; (v) type of thiopurine; (vi) duration of treatment ( $<$ or $\geq 8$ weeks); (vii) concomitant medication; and (viii) provided vs. calculated means.

An optimal cut-off for leukopenia was calculated according to the method described by Steinhauser [39].

\section{Results}

\subsection{Bibliographic Search and Study Selection}

The adopted study selection strategy is detailed in Figure 1. From the initial 21,240 reports, after duplicates removal, initial screening and full-text retrieval and analysis, 127 articles met the criteria for qualitative synthesis. Of these, 72 had appropriate data to be included in the meta-analysis. 


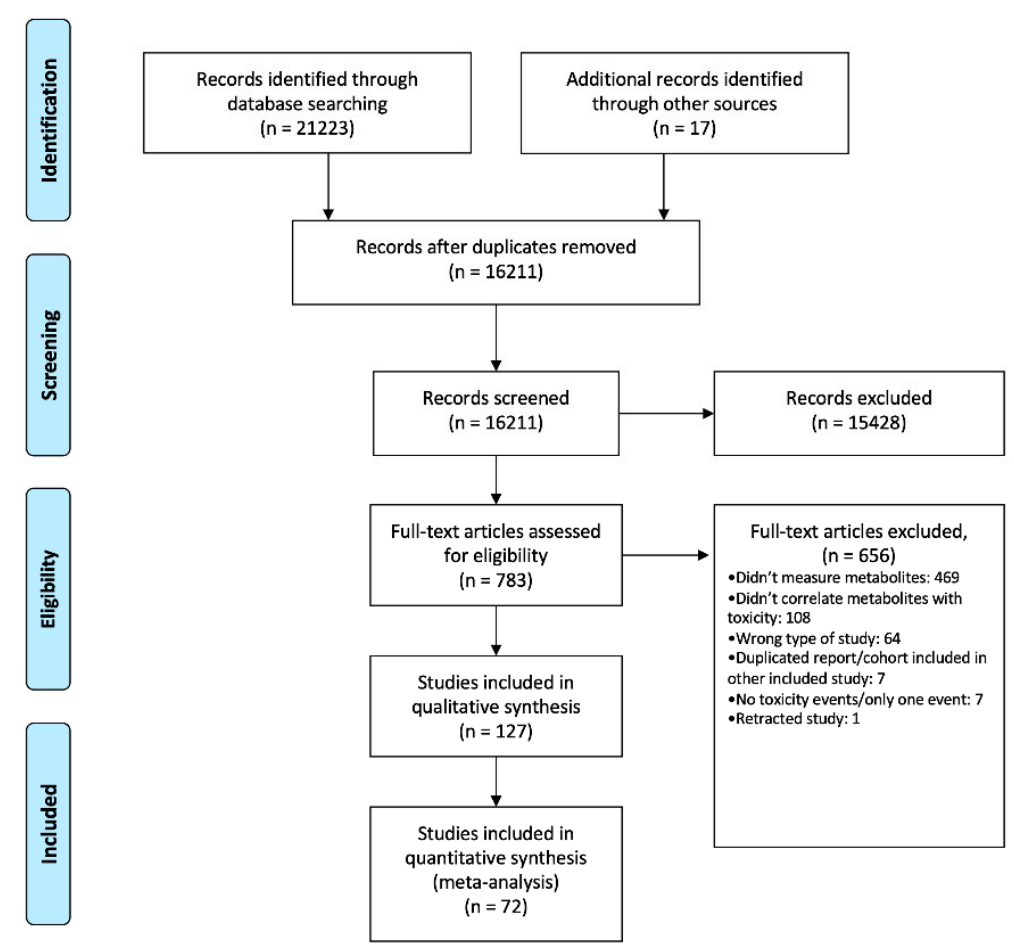

Figure 1. Bibliographic search and study selection-Preferred Reporting Items for Systematic Reviews and Meta-Analyses (PRISMA) flow diagram.

\subsection{Description of the Studies}

The details of the 72 studies included in the meta-analysis are presented in Table 1 and Table S1. The 72 included studies were published between 1983 and 2018. Most studies $(n=60,83 \%)$ were from Western countries. The distribution between pediatric $(n=29,40 \%)$ and adult cohorts $(n=30,42 \%)$ was balanced. Most studies included patients with IBD only $(n=42,58 \%)$, followed by ALL $(n=16,22 \%)$. In the majority of reports, patients were treated with conventional thiopurines ( $n=63,89 \%)$ and were being treated for 8 weeks or longer at the time of the first metabolite measurement $(n=35,49 \%)$. Most reports included patients concomitantly treated with other immunosuppressants or chemotherapy drugs. In 31 studies, data of patients without those concomitant drugs were provided and was used in our meta-analysis; still, steroids, mesalamine and/or antibiotics were allowed in most.

The measurement of metabolites followed the procedures described by Lennard $[2,30]$ or were converted to approximate values in 33 studies, the methodology described by Dervieux-Boulieu [3] in 16 studies and the method described by Erdmann et al. [32] in 6 studies. In nine reports the measurements were performed in Prometheus Lab with a proprietary method. The study by Fangbin et al., (2016) [40] used the Dervieux-Boulieu methodology for measurement of 6-TGN and the Lennard method for 6-MMPR. Data for both metabolites (6-TGN and 6-MMPR) were available in 21 studies; 41 studies provided only usable data for 6-TGN and 10 studies for 6-MMPR. In six studies, it was also possible to evaluate the 6-MMPR/6-TGN ratio. We could identify some discrepancies concerning the nomenclature of methylated metabolites. The most common terms were 6-MMPR, 6-MMP, and methylated thioinosine monophosphate (meTIMP). In the methods commonly used for thiopurines' metabolites measurement, the methylated metabolites are hydrolyzed back to 4-amino-5-methylthiocarbonyl imidazole, a common derivative, rendering them indistinguishable. Therefore, both Dervieux and Lennard assays measure the sum of all methylated metabolites [13,41]. In this paper, the term used to describe these metabolites is 6-MMPR. 
Table 1. Description of the 72 studies included in the meta-analysis.

\begin{tabular}{|c|c|c|c|c|c|c|c|c|c|c|}
\hline Study & $\begin{array}{c}\text { Study } \\
\text { Design }\end{array}$ & Study Population & $\begin{array}{c}\text { Population with } \\
\text { Evaluation of } \\
\text { Metabolites and } \\
\text { Toxicity (If Different } \\
\text { from Total Number) }\end{array}$ & Disease & $\begin{array}{l}\text { Treatment Regimen } \\
\text { and Duration }\end{array}$ & $\begin{array}{l}\text { Concomitant } \\
\text { Medication }\end{array}$ & $\begin{array}{c}\text { Measured } \\
\text { Metabolites }\end{array}$ & $\begin{array}{l}\text { Measurements Per } \\
\text { Patient (If Multiple, } \\
\text { Used Value) }\end{array}$ & Method & $\begin{array}{c}\text { QAT Score } \\
(\%)\end{array}$ \\
\hline $\begin{array}{l}\text { Adam de Beaumais et al. } \\
\text { Br J Clin Pharm } 2011 \\
\text { [44] }\end{array}$ & 1., & 66 patients, pediatric & Same & Leukemia & $\begin{array}{c}\text { 6-MP } \\
>4 \text { weeks }\end{array}$ & Methotrexate & 6-MMPR & $\begin{array}{l}\text { Multiple (average of } \\
\text { all samples per } \\
\text { patient) }\end{array}$ & $\begin{array}{l}\text { Dervieux } \\
\text { and Boulieu }\end{array}$ & 67.8 \\
\hline $\begin{array}{l}\text { Almer et al., Dig Liver } \\
\text { Dis } 2009 \text { [45] }\end{array}$ & Prospective & 23 patients, adult & Same & IBD & $\begin{array}{l}\text { 6-TG } \\
\text { From the start } \\
\text { (timeline not } \\
\text { discriminated) }\end{array}$ & $\begin{array}{l}\text { Mesalazine, steroids } \\
\text { and antibiotics. }\end{array}$ & 6-TGN & $\begin{array}{l}\text { Multiple (maximum } \\
\text { value of metabolites) }\end{array}$ & $\begin{array}{l}\text { Lennard and } \\
\text { Singleton }\end{array}$ & 40.5 \\
\hline $\begin{array}{l}\text { Alvarez Beltran et al., } \\
\text { An Pediatr } 2009 \text { [46] }\end{array}$ & Retrospective & 107 patients, pediatric & 18 patients & $\begin{array}{l}\text { IBD and } \\
\text { AIH }\end{array}$ & $\begin{array}{l}\text { AZA } \\
>2 \text { months }\end{array}$ & NS & $\begin{array}{c}\text { 6-TGN and } \\
\text { 6-MMPR }\end{array}$ & NS & NS & 42.9 \\
\hline $\begin{array}{c}\text { Andoh et al., J } \\
\text { Gastroenterol Hepatol } \\
2008 \text { [47] }\end{array}$ & Retrospective & 83 patients, adult & Same & IBD & $\begin{array}{l}\mathrm{AZA}+6-\mathrm{MP} \\
>4 \text { months }\end{array}$ & Mesalazine & 6-TGN & NS & Erdmann & 41.7 \\
\hline $\begin{array}{l}\text { Armstrong et al., } \\
\text { Aliment Pharmacol } \\
\text { Ther } 2011 \text { [48] }\end{array}$ & Retrospective & 70 patients, pediatric & Same & IBD & $\begin{array}{l}\text { AZA + 6-MP } \\
>3 \text { months }\end{array}$ & Mesalazine & 6-TGN & Multiple (NS) & $\begin{array}{l}\text { Dervieux } \\
\text { and Boulieu }\end{array}$ & 38.1 \\
\hline $\begin{array}{c}\text { Ban et al., J } \\
\text { Gastroenterol } 2010 \\
\text { [49] }\end{array}$ & Prospective & $\begin{array}{c}279 \text { patients, pediatric } \\
\text { and adult }\end{array}$ & 130 patients & IBD & $\begin{array}{c}\mathrm{AZA}+6-\mathrm{MP} \\
\mathrm{NS}\end{array}$ & Mesalazine & 6-TGN & NS & Erdmann & 54.7 \\
\hline $\begin{array}{l}\text { Banerjee et al., J } \\
\text { Pediatr Gastroenterol } \\
\text { Nutr } 2006 \text { [50] }\end{array}$ & Retrospective & 101 patients, pediatric & 64 patients & IBD & $\begin{array}{c}\mathrm{AZA}+6-\mathrm{MP} \\
>6 \text { months }\end{array}$ & $\begin{array}{l}\text { Mesalazine, steroids, } \\
\text { antibiotics and } \\
\text { infliximab }\end{array}$ & 6-MMP & $\begin{array}{l}\text { Multiple (evaluation } \\
\text { per-sample) }\end{array}$ & Prometheus & 65.5 \\
\hline $\begin{array}{l}\text { Belaiche et al., Scand J } \\
\text { Gastroenterol } 2001 \\
\text { [51] }\end{array}$ & Prospective & 28 patients, adult & Same & IBD & $\begin{array}{c}\mathrm{AZA}+6-\mathrm{MP} \\
>3 \text { months }\end{array}$ & Steroids & 6-TGN & Single & $\begin{array}{l}\text { Lennard and } \\
\text { Singleton }\end{array}$ & 52.4 \\
\hline $\begin{array}{c}\text { Bergan et al., } \\
\text { Transplantation } 1994 \\
\text { [52] }\end{array}$ & Prospective & $\begin{array}{l}65 \text { patients, pediatric } \\
\text { and adult }\end{array}$ & 62 patients & $\begin{array}{c}\text { Renal } \\
\text { transplant }\end{array}$ & $\begin{array}{c}\text { AZA } \\
\text { Initial } 40 \text { days }\end{array}$ & $\begin{array}{l}\text { Ciclosporin and } \\
\text { steroids }\end{array}$ & 6-TGN & $\begin{array}{l}\text { Multiple (division in } 2 \\
\text { groups: patients with } \\
\text { all 6-TGN below } \\
\text { threshold, and } \\
\text { patients with at least } \\
\text { one 6-TGN measure } \\
\text { above threshold) }\end{array}$ & Lennard & 67.8 \\
\hline $\begin{array}{l}\text { Berkovitch et al., Med } \\
\text { Pediatr Oncol } 1996\end{array}$ & Retrospective & 29 patients, pediatric & 8 patients & Leukemia & $\begin{array}{l}\text { 6-MP } \\
\text { NS }\end{array}$ & Chemotherapy & 6-MMPR & Single & $\begin{array}{l}\text { Lennard and } \\
\text { Singleton }\end{array}$ & 40.5 \\
\hline $\begin{array}{c}\text { Boulieu et al., Br J } \\
\text { Clin Pharm } 1997 \text { [54] }\end{array}$ & Prospective & 47 patients, adult & Same & Transplant & $\underset{>3 \text { months }}{\mathrm{AZA}}$ & $\begin{array}{l}\text { Cyclosporine and } \\
\text { steroids }\end{array}$ & 6-TGN & Single & $\begin{array}{l}\text { Dervieux } \\
\text { and Boulieu }\end{array}$ & 39.3 \\
\hline $\begin{array}{l}\text { Boulieu et al., Adv } \\
\text { Exp Med Biol } 2000 \\
\text { [55] }\end{array}$ & Prospective & 27 patients, adult & Same & Transplant & $\begin{array}{c}\text { AZA } \\
>3 \text { months }\end{array}$ & $\begin{array}{l}\text { Steroids and } \\
\text { Cyclosporine }\end{array}$ & $\begin{array}{c}\text { 6-TGN and } \\
\text { 6-MMPR }\end{array}$ & Single & $\begin{array}{l}\text { Dervieux } \\
\text { and Boulieu }\end{array}$ & 45.2 \\
\hline $\begin{array}{c}\text { Broekman et al., } \\
\text { Aliment Pharm Ther } \\
2017 \text { [56] }\end{array}$ & Prospective & 695 patients, adult & 301 patients & IBD & $\begin{array}{c}\text { AZA + 6-MP } \\
\text { Week } 8\end{array}$ & $\begin{array}{l}\text { Mesalazine, steroids, } \\
\text { biologics }\end{array}$ & $\begin{array}{c}\text { 6-TGN and } \\
\text { 6-MMPR }\end{array}$ & Single & $\begin{array}{l}\text { Lennard and } \\
\text { Singleton }\end{array}$ & 76.2 \\
\hline
\end{tabular}


Table 1. Cont

\begin{tabular}{|c|c|c|c|c|c|c|c|c|c|c|}
\hline Study & $\begin{array}{c}\text { Study } \\
\text { Design }\end{array}$ & Study Population & $\begin{array}{l}\text { Population with } \\
\text { Evaluation of } \\
\text { Metabolites and } \\
\text { Toxicity (If Different } \\
\text { from Total Number) }\end{array}$ & Disease & $\begin{array}{l}\text { Treatment Regimen } \\
\text { and Duration }\end{array}$ & $\begin{array}{l}\text { Concomitant } \\
\text { Medication }\end{array}$ & $\begin{array}{c}\text { Measured } \\
\text { Metabolites }\end{array}$ & $\begin{array}{l}\text { Measurements Per } \\
\text { Patient (If Multiple, } \\
\text { Used Value) }\end{array}$ & Method & $\begin{array}{c}\text { QAT Score } \\
(\%)\end{array}$ \\
\hline $\begin{array}{c}\text { Chapdelaine et al., J } \\
\text { Clin Rheumatol } 2017 \\
\text { [57] }\end{array}$ & Retrospective & 71 patients, adult & Same & $\begin{array}{l}\text { Rheumatologic } \\
\text { disorders }\end{array}$ & $\begin{array}{l}\text { AZA } \\
\text { NS }\end{array}$ & NS & $\begin{array}{l}\text { 6-TGN and } \\
\text { 6-MMPR }\end{array}$ & Multiple (NS) & $\begin{array}{l}\text { Lennard and } \\
\text { Singleton }\end{array}$ & 65.5 \\
\hline $\begin{array}{c}\text { Chrzanowska et al., } \\
\text { Eur J Pharm Sci } 1999 \\
{[58]}\end{array}$ & Prospective & 19 patients, pediatric & Same & Leukemia & $\begin{array}{c}\text { 6-MP } \\
>1 \text { month }\end{array}$ & Methotrexate & $\begin{array}{l}\text { 6-TGN and } \\
\text { 6-MMPR }\end{array}$ & Single & $\begin{array}{l}\text { Lennard and } \\
\text { Singleton }\end{array}$ & 63.1 \\
\hline $\begin{array}{c}\text { Chrzanowska et al., } \\
\text { Ther Drug Monit } 1999 \\
\text { [59] }\end{array}$ & Prospective & $\begin{array}{l}37 \text { patients, pediatric } \\
\text { and adult }\end{array}$ & Same & $\begin{array}{l}\text { Transplant } \\
\text { and } \\
\text { glomerulonephriti }\end{array}$ & $\begin{array}{c}\text { AZA } \\
>1 \text { month }\end{array}$ & $\begin{array}{l}\text { Cyclosporine and } \\
\text { steroids }\end{array}$ & 6-TGN & Single & $\begin{array}{l}\text { Lennard and } \\
\text { Singleton }\end{array}$ & 46.4 \\
\hline $\begin{array}{c}\text { Cuffari et al., Gut } 1996 \\
\text { [60] }\end{array}$ & Prospective & 25 patients, pediatric & Same & IBD & $\begin{array}{c}\text { 6-MP } \\
>4 \text { months }\end{array}$ & Low-dose steroids & $\begin{array}{l}\text { 6-TGN and } \\
\text { 6-MMP }\end{array}$ & Single & $\begin{array}{l}\text { Lennard and } \\
\text { Singleton }\end{array}$ & 61.9 \\
\hline $\begin{array}{l}\text { Cuffari et al., Dig Dis } \\
\text { Sci } 2004 \text { [61] }\end{array}$ & Prospective & 46 patients, adult & Same & IBD & $\begin{array}{l}\text { AZA } \\
\text { NS }\end{array}$ & $\begin{array}{l}\text { Mesalazine and } \\
\text { "other medications" } \\
\text { allowed (NS) }\end{array}$ & 6-TGN & NS & $\begin{array}{l}\text { Lennard and } \\
\text { Singleton }\end{array}$ & 60.7 \\
\hline $\begin{array}{c}\text { Dassopoulos et al., } \\
\text { Aliment Pharmacol } \\
\text { Ther } 2014 \text { [62] }\end{array}$ & Prospective & $\begin{array}{l}50 \text { patients, pediatric } \\
\text { and adult }\end{array}$ & Same & IBD & $\begin{array}{c}\text { AZA } \\
\text { Week } 4 \text { to week } 44\end{array}$ & $\begin{array}{c}\text { Steroids; other } \\
\text { immunosuppressants } \\
\text { not allowed }\end{array}$ & 6-TGN & NS & Prometheus & 71.4 \\
\hline $\begin{array}{l}\text { De Boer et al., World J } \\
\text { Gastroenterol } 2005 \\
\text { [63] }\end{array}$ & Retrospective & 95 patients, adult & 55 patients & IBD & $\begin{array}{c}\text { TG } \\
>4 \text { weeks }\end{array}$ & $\begin{array}{c}\text { Other } \\
\text { immunosuppressants } \\
\text { not allowed } \\
\text { (cyclosporine, } \\
\text { infliximab, } \\
\text { methotrexate, } \\
\text { thalidomide) }\end{array}$ & 6-TGN & Single & $\begin{array}{l}\text { Lennard and } \\
\text { Singleton }\end{array}$ & 41.7 \\
\hline $\begin{array}{c}\text { Derijks et al., Eur J } \\
\text { Gastroen Hepat } 2003 \\
\text { [64] }\end{array}$ & Prospective & 32 patients, adult & Same & IBD & $\begin{array}{c}\text { 6-TG } \\
\text { Week } 1 \text { to week } 8\end{array}$ & $\begin{array}{c}\text { Other } \\
\begin{array}{c}\text { immunosuppressants } \\
\text { not allowed }\end{array}\end{array}$ & 6-TGN & $\begin{array}{l}\text { Multiple (correlation } \\
\text { per event) }\end{array}$ & $\begin{array}{l}\text { Lennard and } \\
\text { Singleton }\end{array}$ & 51.2 \\
\hline $\begin{array}{l}\text { Derijks et al., Ther } \\
\text { Drug Monit 2004 [65] }\end{array}$ & Prospective & 30 patients, adult & 17 patients & IBD & $\begin{array}{c}\text { 6-MP } \\
\text { Week } 1 \text { to week } 8\end{array}$ & $\begin{array}{c}\text { Mesalazine; other } \\
\text { immunosuppressants } \\
\text { not allowed }\end{array}$ & 6-TGN & $\begin{array}{l}\text { Multiple (level of } \\
\text { metabolites at the } \\
\text { time of AE; for the } \\
\text { non-AE group, levels } \\
\text { at week 8) }\end{array}$ & $\begin{array}{l}\text { Lennard and } \\
\text { Singleton }\end{array}$ & 64.3 \\
\hline $\begin{array}{c}\text { Dervieux et al., } \\
\text { Leukemia } 2001 \text { [66] }\end{array}$ & Prospective & 78 patients, pediatric & 25 patients & Leukemia & $\begin{array}{c}\text { 6-MP } \\
\text { At least }>4 \text { weeks }\end{array}$ & Methotrexate & 6-TGN & $\begin{array}{l}\text { Multiple (steady-state } \\
\text { concentration) }\end{array}$ & $\begin{array}{l}\text { Dervieux } \\
\text { and Boulieu }\end{array}$ & 51.2 \\
\hline $\begin{array}{l}\text { Ding et al., Inflamm } \\
\text { Bowel Dis } 2012[67]\end{array}$ & Prospective & $\begin{array}{l}120 \text { patients, pediatric } \\
\text { and adult }\end{array}$ & 104 patients & IBD & $\begin{array}{c}\text { AZA + 6-MP } \\
\text { Week } 8\end{array}$ & $\begin{array}{l}\text { Mesalazine and } \\
\text { infliximab; } \\
\text { methotrexate and } \\
\text { cyclosporine not } \\
\text { allowed }\end{array}$ & 6-TGN & Single & $\begin{array}{l}\text { Dervieux } \\
\text { and Boulieu }\end{array}$ & 76.2 \\
\hline $\begin{array}{c}\text { Dubinsky et al., } \\
\text { Gastroenterology } \\
2000[14]\end{array}$ & Prospective & 92 patients, pediatric & Same & IBD & $\begin{array}{l}\mathrm{AZA}+6-\mathrm{MP} \\
>4 \text { months }\end{array}$ & Mesalazine & 6-MMPR & Multiple (NS) & $\begin{array}{l}\text { Lennard and } \\
\text { Singleton }\end{array}$ & 66.7 \\
\hline $\begin{array}{c}\text { Dubinsky et al., } \\
\text { Gastroenterology } \\
2002[68]\end{array}$ & Retrospective & $\begin{array}{l}51 \text { patients, pediatric } \\
\text { and adult }\end{array}$ & Same & IBD & $\begin{array}{l}\text { AZA + 6-MP } \\
>3 \text { months }\end{array}$ & $\begin{array}{l}\text { Mesalazine and } \\
\text { steroids }\end{array}$ & 6-MMPR & $\begin{array}{l}\text { Multiple (median } \\
\text { values) }\end{array}$ & Prometheus & 70.2 \\
\hline
\end{tabular}


Table 1. Cont

\begin{tabular}{|c|c|c|c|c|c|c|c|c|c|c|}
\hline Study & $\begin{array}{c}\text { Study } \\
\text { Design }\end{array}$ & Study Population & $\begin{array}{l}\text { Population with } \\
\text { Evaluation of } \\
\text { Metabolites and } \\
\text { Toxicity (If Different } \\
\text { from Total Number) }\end{array}$ & Disease & $\begin{array}{l}\text { Treatment Regimen } \\
\text { and Duration }\end{array}$ & $\begin{array}{l}\text { Concomitant } \\
\text { Medication }\end{array}$ & $\begin{array}{c}\text { Measured } \\
\text { Metabolites }\end{array}$ & $\begin{array}{l}\text { Measurements Per } \\
\text { Patient (If Multiple, } \\
\text { Used Value) }\end{array}$ & Method & $\begin{array}{c}\text { QAT Score } \\
(\%)\end{array}$ \\
\hline $\begin{array}{c}\text { Dubinsky et al., } \\
\text { Gastroenterology } \\
2003 \text { [69] }\end{array}$ & Retrospective & $\begin{array}{l}111 \text { patients, pediatric } \\
\text { and adult }\end{array}$ & Same & IBD & $\begin{array}{l}\text { 6-TG } \\
1 \text { to } 28 \text { months }\end{array}$ & $\begin{array}{l}\text { Steroids, mesalazine } \\
\text { and infliximab }\end{array}$ & 6-TGN & NS & Prometheus & 54.8 \\
\hline $\begin{array}{l}\text { Fangbin et al., } \\
\text { Medicine } 2016 \text { [40] }\end{array}$ & Prospective & 132 patients, adult & Same & IBD & $\begin{array}{c}\text { AZA } \\
\text { Week } 1 \text { to week } 48\end{array}$ & $\begin{array}{l}\text { Mesalazine and } \\
\text { infliximab }\end{array}$ & 6-TGN & $\begin{array}{l}\text { Multiple (maximum } \\
\text { tgn at the time of AE) } \\
\text { For optimal cut-off } \\
\text { calculus, all } 471 \\
\text { samples were used }\end{array}$ & $\begin{array}{c}\text { Dervieux } \\
\text { and Boulieu } \\
\text { for 6-TGN } \\
\text { and Lennard } \\
\text { and } \\
\text { Singleton for } \\
\text { 6-MMP }\end{array}$ & 64.3 \\
\hline $\begin{array}{c}\text { Fei et al., Front } \\
\text { Pharmacol } 2018 \text { [70] }\end{array}$ & Retrospective & 87 patients, adult & Same & $\begin{array}{l}\text { Multiple } \\
\text { (NS) }\end{array}$ & $\begin{array}{c}\text { AZA } \\
>2 \text { months }\end{array}$ & $\begin{array}{c}\text { Medications } \\
\text { interfering with } \\
\text { metabolite levels } \\
\text { and/or causing } \\
\text { leukopenia were } \\
\text { excluded } \\
\text { (cycloscporine, } \\
\text { tacrolimus, } \\
\text { mesalazine, } \\
\text { allopurinol, diuretics) }\end{array}$ & 6-TGN & Single & $\begin{array}{l}\text { Dervieux } \\
\text { and Boulieu }\end{array}$ & 70.3 \\
\hline $\begin{array}{c}\text { Feng et al., J } \\
\text { Gastroenterol Hepatol } \\
2018 \text { [71] }\end{array}$ & Retrospective & 252 patients, adult & Same & IBD & $\begin{array}{c}\text { AZA } \\
>3 \text { months }\end{array}$ & $\begin{array}{l}\text { Mesalazine and } \\
\text { antibiotics; biologics, } \\
\text { thalidomide and } \\
\text { steroids not allowed }\end{array}$ & 6-TGN & $\begin{array}{l}\text { Multiple (evaluation } \\
\text { per-sample) }\end{array}$ & $\begin{array}{l}\text { Dervieux } \\
\text { and Boulieu }\end{array}$ & 73.8 \\
\hline $\begin{array}{l}\text { Ferucci et al., Can J } \\
\text { Gastroenterol } 2011 \\
\text { [72] }\end{array}$ & Retrospective & 71 patients, adult & 48 patients & $\mathrm{AIH}$ & $\begin{array}{l}\text { AZA } \\
\text { NS }\end{array}$ & NS & $\begin{array}{l}\text { 6-TGN and } \\
\text { 6-MMPR }\end{array}$ & $\begin{array}{l}\text { Multiple (most recent } \\
\text { value available) }\end{array}$ & Prometheus & 70.2 \\
\hline $\begin{array}{l}\text { Ganping et al., Int J } \\
\text { Pharmacol } 2008 \text { [73] }\end{array}$ & Prospective & 10 patients, pediatric & Same & Leukemia & $\begin{array}{c}\text { 6-MP } \\
>2 \text { months }\end{array}$ & Methotrexate & 6-TGN & $\begin{array}{c}\text { Multiple (level of } \\
\text { metabolites measured } \\
7 \text { days before } \\
\text { laboratorial } \\
\text { evaluation of } \mathrm{AE} \text { ) }\end{array}$ & $\begin{array}{l}\text { Lennard and } \\
\text { Singleton }\end{array}$ & 39.3 \\
\hline $\begin{array}{c}\text { Gardiner et al., Clin } \\
\text { Gastroenterol Hepatol } \\
2008 \text { [74] }\end{array}$ & Prospective & $\begin{array}{c}69 \text { patients, }>16 \text { years } \\
\text { old }\end{array}$ & 61 patients & IBD & $\begin{array}{c}\text { AZA + 6-MP } \\
\text { Month } 1 \text { to month } 9\end{array}$ & $\begin{array}{l}\text { No patient was } \\
\text { excluded based on } \\
\text { concomitant } \\
\text { medication; } \\
\text { concomitant drugs NS }\end{array}$ & $\begin{array}{l}\text { 6-TGN and } \\
\text { 6-MMPR }\end{array}$ & $\begin{array}{l}\text { Multiple (level of } \\
\text { metabolites within } 2 \\
\text { days of stopping } \\
\text { treatment in the AE } \\
\text { group; for the non-AE } \\
\text { group, values at } \\
\text { month 1) }\end{array}$ & $\begin{array}{l}\text { Dervieux } \\
\text { and Boulieu }\end{array}$ & 66.7 \\
\hline $\begin{array}{c}\text { Gupta et al., J Pediatr } \\
\text { Gastroenterol Nutr } \\
2001 \text { [75] }\end{array}$ & Retrospective & 101 patients, pediatric & Same & IBD & $\begin{array}{c}\mathrm{AZA}+6-\mathrm{MP} \\
>4 \text { months }\end{array}$ & NS & $\begin{array}{l}\text { 6-TGN and } \\
\text { 6-MMPR }\end{array}$ & Multiple (NS) & Prometheus & 52.4 \\
\hline $\begin{array}{c}\text { Halonen et al., Pediatr } \\
\text { Blood Cancer } 2006 \\
\text { [76] }\end{array}$ & Prospective & 16 patients, pediatric & Same & Leukemia & $\begin{array}{c}\text { 6-MP } \\
\text { NS }\end{array}$ & Chemotherapy & 6-TGN & $\begin{array}{l}\text { Multiple (average of } \\
\text { all samples per } \\
\text { patient) }\end{array}$ & Bruunshuus & 59.5 \\
\hline
\end{tabular}


Table 1. Cont

\begin{tabular}{|c|c|c|c|c|c|c|c|c|c|c|}
\hline Study & $\begin{array}{c}\text { Study } \\
\text { Design }\end{array}$ & Study Population & $\begin{array}{c}\text { Population with } \\
\text { Evaluation of } \\
\text { Metabolites and } \\
\text { Toxicity (If Different } \\
\text { from Total Number) }\end{array}$ & Disease & $\begin{array}{l}\text { Treatment Regimen } \\
\text { and Duration }\end{array}$ & $\begin{array}{l}\text { Concomitant } \\
\text { Medication }\end{array}$ & $\begin{array}{c}\text { Measured } \\
\text { Metabolites }\end{array}$ & $\begin{array}{l}\text { Measurements Per } \\
\text { Patient (If Multiple, } \\
\text { Used Value) }\end{array}$ & Method & $\begin{array}{c}\text { QAT Score } \\
(\%)\end{array}$ \\
\hline $\begin{array}{l}\text { Hande et al., Inflamm } \\
\text { Bowel Dis } 2006 \text { [77] }\end{array}$ & Retrospective & $\begin{array}{l}126 \text { patients, pediatric } \\
\text { and adult }\end{array}$ & 121 patients & IBD & $\begin{array}{l}\mathrm{AZA}+6-\mathrm{MP} \\
>3 \text { months }\end{array}$ & $\begin{array}{c}\text { Mesalazine; steroids, } \\
\text { infliximab and other } \\
\text { immunosuppressants } \\
\text { not allowed }\end{array}$ & $\begin{array}{c}\text { 6-TGN and } \\
\text { 6-MMPR }\end{array}$ & $\begin{array}{l}\text { Multiple (most recent } \\
\text { values) }\end{array}$ & Prometheus & 73.8 \\
\hline $\begin{array}{l}\text { Heerasing et al., } \\
\text { Intern Med J } 2016 \text { [78] }\end{array}$ & Retrospective & 67 patients, NS & Same & IBD & $\begin{array}{c}\mathrm{AZA}+6-\mathrm{MP} \\
\mathrm{NS}\end{array}$ & NS & 6-TGN & NS & NS & 42.9 \\
\hline $\begin{array}{l}\text { Hindorf et al., } \\
\text { Aliment Pharmacol } \\
\text { Ther } 2006 \text { [79] }\end{array}$ & Retrospective & $\begin{array}{l}364 \text { patients, pediatric } \\
\text { and adult }\end{array}$ & 266 patients & IBD & $\begin{array}{c}\mathrm{AZA}+6-\mathrm{MP}+6-\mathrm{TG} \\
\mathrm{NS}\end{array}$ & $\begin{array}{l}\text { Only mesalazine and } \\
\text { steroids }\end{array}$ & $\begin{array}{l}\text { 6-TGN and } \\
\text { 6-MMPR }\end{array}$ & $\begin{array}{l}\text { Multiple (at the time } \\
\text { of } \mathrm{AE} \text {; for the non- } \mathrm{AE} \\
\text { group, last result } \\
\text { available) }\end{array}$ & $\begin{array}{l}\text { Lennard and } \\
\text { Singleton }\end{array}$ & 81.0 \\
\hline $\begin{array}{l}\text { Hindorf et al., Gut et } \\
\text { al. } 2006 \text { [80] }\end{array}$ & Prospective & 60 patients, adult & 54 patients & IBD & $\begin{array}{c}\text { AZA + 6-MP } \\
\text { Week } 1 \text { to week } 20\end{array}$ & $\begin{array}{l}\text { Mesalazine, steroids, } \\
\text { infliximab }\end{array}$ & $\begin{array}{l}\text { 6-TGN and } \\
\text { 6-MMPR }\end{array}$ & $\begin{array}{l}\text { Multiple (maximum } \\
\text { value of metabolites) }\end{array}$ & $\begin{array}{l}\text { Lennard and } \\
\text { Singleton }\end{array}$ & 59.5 \\
\hline $\begin{array}{l}\text { Innocenti et al., Ther } \\
\text { Drug Monit } 2000 \text { [81] }\end{array}$ & Prospective & 19 patients, pediatric & Same & Leukemia & $\begin{array}{c}\text { 6-MP } \\
>3 \text { months }\end{array}$ & Chemotherapy & 6-TGN & $\begin{array}{l}\text { Multiple (evaluation } \\
\text { per-sample) }\end{array}$ & $\begin{array}{l}\text { Lennard and } \\
\text { Singleton }\end{array}$ & 65.5 \\
\hline $\begin{array}{l}\text { Kopylov et al., J } \\
\text { Pediatr Gastroenterol } \\
\text { Nutr } 2014 \text { [82] }\end{array}$ & Prospective & 237 patients, pediatric & Same & IBD & $\begin{array}{l}\mathrm{AZA}+6-\mathrm{MP} \\
>3 \text { months }\end{array}$ & $\begin{array}{l}\text { Mesalazine and } \\
\text { steroids; methotrexate } \\
\text { and biologics not } \\
\text { allowed }\end{array}$ & 6-MMPR & $\begin{array}{l}\text { Multiple (evaluation } \\
\text { per-sample) }\end{array}$ & $\begin{array}{l}\text { Lennard and } \\
\text { Singleton }\end{array}$ & 63.1 \\
\hline $\begin{array}{l}\text { Lancaster et al., Br J } \\
\text { Haematol } 1998 \text { [83] }\end{array}$ & Prospective & 46 patients, pediatric & 37 patients & Leukemia & $\begin{array}{c}\text { 6-MP + 6-TG } \\
\text { Measurements } \\
\text { available from at least } \\
\text { week } 3 \text { (not } \\
\text { mentioned if for all } \\
\text { patients) }\end{array}$ & Chemotherapy & 6-TGN & $\begin{array}{l}\text { Multiple (earliest } \\
\text { essay) }\end{array}$ & $\begin{array}{l}\text { Lennard and } \\
\text { Singleton }\end{array}$ & 53.6 \\
\hline $\begin{array}{l}\text { Lee at al., Inflamm } \\
\text { Bowel Dis } 2015 \text { [84] }\end{array}$ & Retrospective & 137 patients, pediatric & Same & IBD & $\begin{array}{l}\text { AZA } \\
>2 \text { months }\end{array}$ & $\begin{array}{l}\text { Mesalazine, steroids, } \\
\text { infliximab } \\
\text { Steroids and }\end{array}$ & 6-TGN & $\begin{array}{l}\text { Multiple (evaluation } \\
\text { per-sample) }\end{array}$ & $\begin{array}{l}\text { Dervieux } \\
\text { and Boulieu }\end{array}$ & 63.1 \\
\hline $\begin{array}{c}\text { Lee et al., PLoS One } \\
2017 \text { [85] }\end{array}$ & Retrospective & 165 patients, adult & Same & IBD & $\begin{array}{l}\mathrm{AZA}+6-\mathrm{MP} \\
>3 \text { months }\end{array}$ & $\begin{array}{l}\text { mesalazine; patients } \\
\text { using anti-TNF were } \\
\text { excluded }\end{array}$ & $\begin{array}{c}\text { 6-TGN and } \\
\text { 6-MMPR }\end{array}$ & NS & $\begin{array}{l}\text { Dervieux } \\
\text { and Boulieu }\end{array}$ & 67.8 \\
\hline $\begin{array}{l}\text { Lennard et al., } \mathrm{Br} \mathrm{J} \\
\text { Clin Pharm } 1983 \text { [86] }\end{array}$ & Prospective & 22 patients, pediatric & Same & Leukemia & $\begin{array}{c}\text { 6-MP } \\
>4 \text { weeks }\end{array}$ & Chemotherapy & 6-TGN & $\begin{array}{c}\text { Multiple (level of } \\
\text { metabolites measured } \\
14 \text { days before } \\
\text { laboratorial } \\
\text { evaluation) }\end{array}$ & $\begin{array}{l}\text { Lennard and } \\
\text { Singleton }\end{array}$ & 70.2 \\
\hline $\begin{array}{l}\text { Lennard et al., Br J } \\
\text { Clin Pharm } 1984 \text { [87] }\end{array}$ & Prospective & 54 patients, NS & 46 patients & Transplant & $\begin{array}{c}\text { AZA } \\
>6 \text { months }\end{array}$ & Steroids & 6-TGN & $\begin{array}{l}\text { Multiple (evaluation } \\
\text { per-sample) }\end{array}$ & $\begin{array}{l}\text { Lennard and } \\
\text { Singleton }\end{array}$ & 51.2 \\
\hline $\begin{array}{l}\text { Lennard et al., Lancet } \\
1990 \text { [88] }\end{array}$ & Retrospective & 225 patients, pediatric & 82 patients & Leukemia & $\begin{array}{c}\text { 6-MP } \\
>2 \text { months }\end{array}$ & Chemotherapy & 6-TGN & Single & $\begin{array}{l}\text { Lennard and } \\
\text { Singleton }\end{array}$ & 46.4 \\
\hline $\begin{array}{l}\text { Lennard et al., Clin } \\
\text { Pharm Ther } 2006 \text { [89] }\end{array}$ & Prospective & $\begin{array}{l}1492 \text { patients, } \\
\text { pediatric }\end{array}$ & 134 patients & Leukemia & $\begin{array}{c}\text { TG } \\
>7 \text { days }\end{array}$ & Chemotherapy & 6-TGN & Single & $\begin{array}{l}\text { Lennard and } \\
\text { Singleton }\end{array}$ & 67.8 \\
\hline $\begin{array}{l}\text { Lilleyman et al., Br J } \\
\text { Cancer } 1984 \text { [90] }\end{array}$ & Prospective & 22 patients, pediatric & Same & Leukemia & $\begin{array}{c}\text { 6-MP } \\
>7 \text { months }\end{array}$ & Chemotherapy & 6-TGN & $\begin{array}{l}\text { Multiple (level of } \\
\text { metabolites measured } \\
14 \text { days before } \\
\text { laboratorial } \\
\text { evaluation) }\end{array}$ & $\begin{array}{l}\text { Lennard and } \\
\text { Singleton }\end{array}$ & 63.1 \\
\hline
\end{tabular}


Table 1. Cont

\begin{tabular}{|c|c|c|c|c|c|c|c|c|c|c|}
\hline Study & $\begin{array}{l}\text { Study } \\
\text { Design }\end{array}$ & Study Population & $\begin{array}{l}\text { Population with } \\
\text { Evaluation of } \\
\text { Metabolites and } \\
\text { Toxicity (If Different } \\
\text { from Total Number) }\end{array}$ & Disease & $\begin{array}{l}\text { Treatment Regimen } \\
\text { and Duration }\end{array}$ & $\begin{array}{l}\text { Concomitant } \\
\text { Medication }\end{array}$ & $\begin{array}{l}\text { Measured } \\
\text { Metabolites }\end{array}$ & $\begin{array}{l}\text { Measurements Per } \\
\text { Patient (If Multiple, } \\
\text { Used Value) }\end{array}$ & Method & $\begin{array}{c}\text { QAT Score } \\
(\%)\end{array}$ \\
\hline $\begin{array}{c}\text { Liu et al., Scand J } \\
\text { Gastroenterol } 2016 \\
\text { [91] }\end{array}$ & Prospective & 69 patients, adult & Same & IBD & $\begin{array}{c}\text { AZA } \\
>3 \text { months }\end{array}$ & $\begin{array}{l}\text { Steroids and } \\
\text { Infliximab }\end{array}$ & 6-TGN & NS & $\begin{array}{l}\text { Dervieux } \\
\text { and Boulieu }\end{array}$ & 69.1 \\
\hline $\begin{array}{c}\text { Meijer et al., J } \\
\text { Gastroenterol Hepatol } \\
2017[37]\end{array}$ & Retrospective & 24 patients, adult & Same & IBD & $\begin{array}{c}\text { AZA + 6-MP } \\
\text { Median 11 weeks } \\
\text { (IQR 6-46) }\end{array}$ & $\begin{array}{l}\text { Steroids; no mention } \\
\text { to additional } \\
\text { medication }\end{array}$ & 6-MMPR & $\begin{array}{l}\text { Multiple (level of } \\
\text { metabolites within } 3 \\
\text { days of AE) }\end{array}$ & $\begin{array}{l}\text { Lennard and } \\
\text { Singleton }\end{array}$ & 50.0 \\
\hline $\begin{array}{l}\text { Meijer et al., Ther } \\
\text { Drug Monit } 2017 \text { [36] }\end{array}$ & Retrospective & 424 patients, adult & Same & $\begin{array}{l}\text { IBD, AIH } \\
\text { and celiac } \\
\text { disease }\end{array}$ & $\begin{array}{c}\mathrm{AZA}+6-\mathrm{MP}+\mathrm{TG} \\
\mathrm{NS}\end{array}$ & - =ет & $\begin{array}{l}\text { 6-TGN and } \\
\text { 6-MMP }\end{array}$ & $\begin{array}{l}\text { Multiple (evaluation } \\
\text { per-sample when } \\
\text { laboratory data } \\
\text { within } 3 \text { days are } \\
\text { available) }\end{array}$ & $\begin{array}{c}\text { Dervieux } \\
\text { and Boulieu } \\
\text { (but } \\
\text { converted to } \\
\text { Lennard by } \\
\text { a factor of } \\
2.6 \text { ) }\end{array}$ & 63.1 \\
\hline $\begin{array}{l}\text { Melaouhia et al., } \\
\text { Therapie } 2013 \text { [92] }\end{array}$ & Prospective & 50 patients, adult & Same & IBD & $\begin{array}{c}\text { AZA } \\
>12 \text { months }\end{array}$ & $\begin{array}{l}\text { Mesalazine and } \\
\text { steroids }\end{array}$ & $\begin{array}{l}\text { 6-TGN and } \\
\text { 6-MMPR }\end{array}$ & Multiple (NS) & $\begin{array}{l}\text { Dervieux } \\
\text { and Boulieu }\end{array}$ & 44.1 \\
\hline $\begin{array}{l}\text { Nguyen et al., Int J } \\
\text { Clin Pharm } 2010 \text { [93] }\end{array}$ & Retrospective & 71 patients, pediatric & Same & IBD & $\begin{array}{c}\text { AZA } \\
>1 \text { year }\end{array}$ & Mesalazine & $\begin{array}{l}\text { 6-TGN and } \\
\text { 6-MMPR }\end{array}$ & $\begin{array}{l}\text { Multiple (evaluation } \\
\text { per-sample) }\end{array}$ & $\begin{array}{l}\text { Dervieux } \\
\text { and Boulieu }\end{array}$ & 38.1 \\
\hline $\begin{array}{c}\text { Nguyen et al., Ther } \\
\text { Drug Monitor } 2010 \\
\text { [94] }\end{array}$ & Retrospective & 28 patients, pediatric & Same & $\mathrm{AIH}$ & $\begin{array}{c}\text { AZA } \\
>3 \text { months }\end{array}$ & Steroids & $\begin{array}{l}\text { 6-TGN and } \\
\text { 6-MMPR }\end{array}$ & Multiple (NS) & $\begin{array}{l}\text { Dervieux } \\
\text { and Boulieu }\end{array}$ & 48.8 \\
\hline $\begin{array}{l}\text { Nygaard et al., Clin } \\
\text { Pharm Ther 2004 [95] }\end{array}$ & Retrospective & 43 patients, pediatric & Same & Leukemia & $\begin{array}{c}\text { 6-MP } \\
>4 \text { weeks }\end{array}$ & Methotrexate & $\begin{array}{l}\text { 6-TGN and } \\
\text { 6-MMPR }\end{array}$ & $\begin{array}{l}\text { Multiple (average of } \\
\text { all samples per } \\
\text { patient) }\end{array}$ & Erdmann & 54.7 \\
\hline $\begin{array}{l}\text { Odahara et al., PLoS } \\
\text { One } 2015 \text { [96] }\end{array}$ & Prospective & 48 patients, adult & Same & IBD & $\begin{array}{l}\text { AZA } \\
\text { NS }\end{array}$ & $\begin{array}{l}\text { Mesalazine and } \\
\text { Infliximab }\end{array}$ & 6-TGN & $\begin{array}{l}\text { Multiple (level of } \\
\text { metabolites at the } \\
\text { time of AE; for the } \\
\text { non-AE group, } \\
\text { mean-value between } \\
\text { weeks } 8 \text { and 52) }\end{array}$ & $\begin{array}{l}\text { Lennard and } \\
\text { Singleton }\end{array}$ & 59.5 \\
\hline $\begin{array}{c}\text { Ohtsuka et al., J } \\
\text { Gastroenterol Hepatol } \\
2010 \text { [97] }\end{array}$ & Retrospective & 51 patients, pediatric & Same & IBD & $\begin{array}{c}\mathrm{AZA}+6-\mathrm{MP} \\
>3 \text { weeks }\end{array}$ & $\begin{array}{l}\text { Mesalazine and } \\
\text { steroids }\end{array}$ & 6-TGN & $\begin{array}{l}\text { Multiple (evaluation } \\
\text { per-sample) }\end{array}$ & Erdmann & 40.5 \\
\hline $\begin{array}{l}\text { Ooi et al., Aliment } \\
\text { Pharm Ther } 2007 \text { [98] }\end{array}$ & Retrospective & 56 patients, pediatric & Same & IBD & $\begin{array}{l}\mathrm{AZA}+6-\mathrm{MP} \\
>1 \text { month }\end{array}$ & $\begin{array}{l}\text { Steroids }>10 \mathrm{mg} / \text { day, } \\
\text { infliximab, tacrolimus, } \\
\text { methotrexate and } \\
\text { cyclosporine not } \\
\text { allowed }\end{array}$ & 6-TGN & $\begin{array}{l}\text { Multiple (evaluation } \\
\text { per-sample) }\end{array}$ & $\begin{array}{l}\text { Lennard and } \\
\text { Singleton }\end{array}$ & 53.6 \\
\hline $\begin{array}{c}\text { Pranzatelli et al., J } \\
\text { Clin Exp Immunol } \\
2017 \text { [99] }\end{array}$ & Retrospective & 10 patients, pediatric & Same & Opsoclonus-n & $\begin{array}{l}\text { yoclonus } \\
>7 \text { months }\end{array}$ & $\begin{array}{l}\text { Adrenocorticotrophic } \\
\text { hormone, intravenous } \\
\text { immunoglobulin and } \\
\text { steroids }\end{array}$ & 6-TGN & Multiple (NS) & Prometheus & 53.6 \\
\hline $\begin{array}{c}\text { Rae et al., J } \\
\text { Neuroimmunol } 2016 \\
{[100]}\end{array}$ & Prospective & 19 patients, adult & Same & $\begin{array}{l}\text { Myasthenia } \\
\text { gravis }\end{array}$ & $\begin{array}{c}\text { AZA } \\
\geq 52 \text { weeks }\end{array}$ & Steroids & $\begin{array}{l}\text { 6-TGN and } \\
\text { 6-MMP }\end{array}$ & NS & $\begin{array}{l}\text { Dervieux } \\
\text { and Boulieu }\end{array}$ & 57.1 \\
\hline
\end{tabular}


Table 1. Cont

\begin{tabular}{|c|c|c|c|c|c|c|c|c|c|c|}
\hline Study & $\begin{array}{c}\text { Study } \\
\text { Design }\end{array}$ & Study Population & $\begin{array}{l}\text { Population with } \\
\text { Evaluation of } \\
\text { Metabolites and } \\
\text { Toxicity (If Different } \\
\text { from Total Number) } \\
\end{array}$ & Disease & $\begin{array}{l}\text { Treatment Regimen } \\
\text { and Duration }\end{array}$ & $\begin{array}{l}\text { Concomitant } \\
\text { Medication }\end{array}$ & $\begin{array}{c}\text { Measured } \\
\text { Metabolites }\end{array}$ & $\begin{array}{l}\text { Measurements Per } \\
\text { Patient (If Multiple, } \\
\text { Used Value) }\end{array}$ & Method & $\begin{array}{c}\text { QAT Score } \\
(\%)\end{array}$ \\
\hline $\begin{array}{c}\text { Sandborn et al., } \\
\text { Gastroenterology } \\
1999[43]\end{array}$ & Prospective & 96 patients, adult & Same & IBD & $\begin{array}{c}\text { AZA } \\
\text { From week } 0.2 \text { to } \\
\text { week } 16\end{array}$ & Steroids & 6-TGN & $\begin{array}{l}\text { Multiple (evaluation } \\
\text { per sample) }\end{array}$ & Erdmann & 88.1 \\
\hline $\begin{array}{l}\text { Schmiegelow et al., } \\
\text { Cancer Chemother } \\
\text { Pharmacol } 1990 \text { [101] }\end{array}$ & Prospective & 31 patients, pediatric & Same & Leukemia & $\begin{array}{c}\text { 6-MP } \\
>5 \text { weeks }\end{array}$ & Chemotherapy & 6-TGN & $\begin{array}{l}\text { Multiple (mean of } \\
\text { measurements) }\end{array}$ & Bruunshuus & 52.4 \\
\hline $\begin{array}{l}\text { Shaye et al., Am J } \\
\text { Gastroenterol } 2007 \\
\text { [102] }\end{array}$ & Retrospective & 173 patients, adult & Same & IBD & $\begin{array}{l}\mathrm{AZA}+6-\mathrm{MP} \\
>1 \text { month }\end{array}$ & Mesalazine & 6-MMPR & NS & Prometheus & 59.5 \\
\hline $\begin{array}{l}\text { Stoneham et al., Br J } \\
\text { Haematol } 2003 \text { [42] }\end{array}$ & Retrospective & 99 patients, pediatric & Same & Leukemia & $\begin{array}{l}\text { 6-MP + TG } \\
\text { Week } 4\end{array}$ & NS & 6-TGN & Single & $\begin{array}{l}\text { Lennard and } \\
\text { Singleton }\end{array}$ & 34.5 \\
\hline $\begin{array}{l}\text { Thomas et al., } \\
\text { Inflamm Bowel Dis } \\
2003 \text { [34] }\end{array}$ & Prospective & 166 patients, adult & 158 patients & IBD & $\begin{array}{l}\mathrm{AZA}+6-\mathrm{MP} \\
>3 \text { months }\end{array}$ & Sulfassalazine & 6-TGN & Single & Erdmann & 63.1 \\
\hline $\begin{array}{c}\text { Wojtuskiewicz et al., } \\
\text { Nucleos Nucleot Nucl } \\
2014 \text { [103] }\end{array}$ & Prospective & $\begin{array}{l}236 \text { patients, pediatric } \\
\text { and adult }\end{array}$ & 41 patients & Leukemia & $\begin{array}{c}\text { 6-MP } \\
\text { Measurements from } \\
\text { week } 25 \text { to } 109\end{array}$ & Chemotherapy & 6-TGN & $\begin{array}{l}\text { Multiple (metabolite } \\
\text { levels at week 25) }\end{array}$ & Keuzenkamp & 63.1 \\
\hline $\begin{array}{c}\text { Wong et al., Aliment } \\
\text { Pharmacol Ther } 2016 \\
\text { [104] }\end{array}$ & Prospective & 270 patients, adult & Same & IBD & $\begin{array}{c}\mathrm{AZA}+6-\mathrm{MP} \\
\text { Week } 1\end{array}$ & $\begin{array}{l}\text { Mesalazine, steroids } \\
\text { and anti-TNF }\end{array}$ & 6-MMPR & Single & $\begin{array}{l}\text { Lennard and } \\
\text { Singleton }\end{array}$ & 82.1 \\
\hline $\begin{array}{l}\text { Wong et al., J Crohn } \\
\text { Colitis } 2017 \text { [105] }\end{array}$ & Prospective & 194 patients, adult & $\begin{array}{l}\text { Data for } 194 \text { patients } \\
\text { available; data from } \\
181 \text { patients were } \\
\text { used in the means } \\
\text { comparison and } \\
\text { pooled OR analyses } \\
\text { (exclusion of patients } \\
\text { using anti-TNF) }\end{array}$ & IBD & $\begin{array}{c}\mathrm{AZA}+6-\mathrm{MP} \\
\text { Week } 1\end{array}$ & $\begin{array}{c}\text { Mesalazine and } \\
\text { steroids; (patients } \\
\text { using anti-TNF were } \\
\text { excluded from means } \\
\text { comparison and } \\
\text { pooled OR analysis; } \\
\text { for calculation of an } \\
\text { optimal cutoff, data } \\
\text { from all patients were } \\
\text { used) }\end{array}$ & $\begin{array}{l}\text { 6-TGN and } \\
\text { 6-MMPR }\end{array}$ & Single & $\begin{array}{l}\text { Lennard and } \\
\text { Singleton }\end{array}$ & 82.1 \\
\hline $\begin{array}{c}\text { Wright et al., Gut } 2004 \\
{[106]}\end{array}$ & Prospective & 159 patients, NS & 123 patients & IBD & $\begin{array}{c}\text { AZA } \\
>4 \text { months }\end{array}$ & $\begin{array}{l}\text { Mesalazine and } \\
\text { steroids }\end{array}$ & 6-MMPR & $\begin{array}{l}\text { Multiple (average of } \\
\text { all samples per } \\
\text { patient) }\end{array}$ & $\begin{array}{l}\text { Lennard and } \\
\text { Singleton }\end{array}$ & 78.6 \\
\hline $\begin{array}{c}\text { Yarur et al., J Clin } \\
\text { Gastroenterol } 2018 \\
\text { [107] }\end{array}$ & Retrospective & 87 patients, adult & Same & IBD & $\begin{array}{c}\mathrm{AZA}+6-\mathrm{MP} \\
>8 \text { weeks }\end{array}$ & $\begin{array}{l}\text { Mesalazine; biologics, } \\
\text { cyclosporine and } \\
\text { tacrolimus not } \\
\text { allowed }\end{array}$ & 6-TGN & $\begin{array}{l}\text { Multiple (nadir } \\
\text { values, median and } \\
\text { peak available; } \\
\text { analysis made with } \\
\text { median) }\end{array}$ & NS & 63.1 \\
\hline $\begin{array}{c}\text { Zochowska et al., } \\
\text { Transplant Proc } 2016 \\
\text { [108] }\end{array}$ & NS & 33 patients, adult & Same & Transplant & $\begin{array}{l}\text { AZA } \\
\text { NS }\end{array}$ & $\begin{array}{l}\text { Calcineurin inhibitors, } \\
\text { steroids }\end{array}$ & $\begin{array}{l}\text { 6-TGN and } \\
\text { 6-MMPR }\end{array}$ & NS & $\begin{array}{l}\text { Other } \\
\text { (description } \\
\text { provided) }\end{array}$ & 51.2 \\
\hline
\end{tabular}

6-MP: 6-mercaptopurine; 6-MMPR: 6 methylmercaptopurine ribonucleotides; 6-TG: 6-thioguanine; 6-TGN: 6-thioguanine nucleotides; AE: adverse events; AIH: autoimmune hepatitis; AZA: azathioprine; IBD: inflammatory bowel disease; NS: non-specified; OR: Odds ratio; QAT: quality assessment tool. 
Multiple metabolites measurements per-patient were performed in 44 studies, whereas on 17 of them only one determination per patient was performed. When multiple determinations were performed, there was a wide variation concerning the value used in the analysis. When described, the used values are specified in Table 1 . The type and definition of toxicities evaluated in each study are detailed in Table S1.

Following Cochrane recommendations, funnel plot asymmetry was only used when at least 10 studies were included [22]; even though in those cases forest plot analysis revealed a considerable amount of variability between studies, funnel plot did not suggest the existence of substantial publication bias.

Regarding quality assessment, the scores ranged from $34.5 \%$ [42] to $88.1 \%$ [43], with a mean value of $58.9 \% \pm 1.46$.

\subsection{Toxicity and Thiopurines' Metabolites}

\subsubsection{Overall Adverse Events}

In some reports, the evaluation of toxicity was performed in broader terms, with the inclusion of all toxicities related to thiopurines in the same group.

\section{6-TGN}

Ten studies evaluated the differences in 6-TGN levels in patients with and without adverse events $[45,46,60,63,69,71,74,79,80,96]$. Overall, the mean 6-TGN levels were not different among patients with and without adverse events, with a pooled difference of $12.41 \mathrm{pmol} / 8 \times 10^{8} \mathrm{RBC}(95 \% \mathrm{CI}$, $-76.18 ; 51.35 ; p=0.70)$. We could notice a significant heterogeneity among these studies $\left(p<0.01 ; \mathrm{I}^{2}=\right.$ $80 \%$ ). In subgroup analysis, age group was partially responsible for studies' inconsistency ( $p=0.04 ; \mathrm{I}^{2}$ $=69 \%$ ). Studies including only adult patients had significantly higher levels of 6-TGN in patients with adverse events (mean difference of $77.62 \mathrm{pmol} / 8 \times 10^{8} \mathrm{RBC}, 95 \%$ CI 38.39; $\left.116.84 ; p<0.01\right)(p=0.66$; $\mathrm{I}^{2}=0 \%$ ) (Figure S1). The use of concomitant medication was not a significant factor accounting for the observed heterogeneity $\left(p=0.84 ; \mathrm{I}^{2}=0 \%\right)$. When the analysis was restricted to studies without concomitant medication (such as chemotherapeutic agents or other immunosuppressors), the mean 6-TGN levels were not different among patients with and without adverse events (mean difference of $\left.23.79 \mathrm{pmol} / 8 \times 10^{8} \mathrm{RBC}, 95 \% \mathrm{CI}-135.21 ; 182.80 ; p=0.77\right)\left(p<0.01 ; \mathrm{I}^{2}=82 \%\right)$.

Four studies provided data to calculate a pooled OR $[46,71,79,96]$ for thresholds of 136.5, 384, 400 and $400 \mathrm{pmol} / 8 \times 10^{8} \mathrm{RBC}$. Patients with 6-TGN levels above the predefined thresholds were almost three times more likely to have adverse events (OR $=2.58,95 \%$ CI 1.36; 4.90; $p<0.01)\left(p=0.33 ; \mathrm{I}^{2}=\right.$ $12 \%)$. When the analysis was restricted to studies using thiopurines in monotherapy, patients with 6-TGN levels above the predefined thresholds were more than three times more likely to experience adverse events (OR 3.52, 95\% CI 1.84; 6.75; $p<0.01)\left(p=0.95 ; \mathrm{I}^{2}=0 \%\right)$.

\section{6-MMPR}

Mean levels of 6-MMPR were significantly higher in patients with adverse events, with a pooled difference of $1184.82 \mathrm{pmol} / 8 \times 10^{8} \mathrm{RBC}(95 \%$ CI 147.00; 2222.64; $p=0.03$ ) (Figure S2) $[46,60,74,79,80]$. The existing heterogeneity $\left(p<0.01 ; \mathrm{I}^{2}=90 \%\right)$ was reduced $\left(p=0.05 ; \mathrm{I}^{2}=62 \%\right)$ when the study by Cuffari et al., 1996 [60] was excluded from the analysis. With the exclusion of this study, the levels of 6-MMPR were similar in patients with and without adverse events. Subgroup analysis did not clarify the origins of inconsistency. Similarly, 6-MMP levels were not different in patients with and without adverse events in those studies using thiopurines in monotherapy, but with considerable heterogeneity between studies (mean difference of $\left.3253.57 \mathrm{pmol} / 8 \times 10^{8} \mathrm{RBC}, 95 \% \mathrm{CI}-2547.48 ; 9054.62 ; p=0.27\right)(p<$ $\left.0.01 ; I^{2}=96 \%\right)$. 


\subsubsection{Myelotoxicity}

"General" Myelotoxicity

In some reports, toxicity combined different aspects of bone marrow suppression, here represented as "general" myelotoxicity.

\section{6-TGN}

In the pooled analysis of four studies, 6-TGN levels in patients with myelotoxicity showed no differences $[46,48,58,80]$, with a mean difference of $169.14 \mathrm{pmol} / 8 \times 10^{8} \mathrm{RBC}(95 \% \mathrm{CI}-69.09 ; 407.37$; $p=0.16)$. However, the heterogeneity was high $\left(p<0.01 ; \mathrm{I}^{2}=93 \%\right)$. In the sub-analysis by duration of thiopurine treatment, only studies including patients with $<8$ weeks of therapy when metabolites were assessed showed numerically higher mean 6-TGN levels in patients with myelotoxicity (Figure S3). A single study reported including patients using thiopurines in monotherapy [48]; in this study there were no differences in 6-TGN levels in patients with and without myelotoxicity, but only patients with more than 3 months of treatment were included.

Three studies provided data to calculate a pooled OR, all of them using a threshold of $450 \mathrm{pmol} / 8 \times$ $10^{8} \mathrm{RBC}[36,46,58]$. The risk of myelotoxicity was almost eight times higher in patients with elevated 6-TGN levels ( $\mathrm{OR}=7.78,95 \%$ CI 1.67; 36.34; $p<0.01)\left(p=0.24 ; \mathrm{I}^{2}=31 \%\right)$. In one of these studies, thiopurines was used as part of a chemotherapy regimen, while in the other two concomitant medications were not specified.

\section{6-MMPR}

In the pooled analysis of four studies, 6-MMPR levels in patients with myelotoxicity were not significantly different $[46,58,79,80]$, with a mean difference of $1601.12 \mathrm{pmol} / 8 \times 10^{8} \mathrm{RBC}(95 \% \mathrm{CI}$ $-559.56 ; 3761.79 ; p=0.15)\left(p=0.08 ; \mathrm{I}^{2}=55 \%\right)$. When the analysis was restricted to studies with IBD patients, 6-MMPR levels were significantly higher in patients with myelotoxicity, with a mean difference of $3529.87 \mathrm{pmol} / 8 \times 10^{8} \mathrm{RBC}(95 \%$ CI $295.65 ; 6764.09 ; p=0.03)$.

Three studies provided data to calculate a pooled OR; two of them used a threshold of 5700 $\mathrm{pmol} / 8 \times 10^{8} \mathrm{RBC}[35,58]$ and the other used a threshold of $11450 \mathrm{pmol} / 8 \times 10^{8} \mathrm{RBC}$ [80]. There was no significant association between 6-MMPR concentrations and overall myelotoxicity (OR 3.83; 95\% CI $0.47 ; 31.37 ; p=0.21)\left(p=0.03, \mathrm{I}^{2}=70 \%\right)$.

\section{6-MMPR/6-TGN Ratio}

The 6-MMPR/6-TGN ratio was not significantly different in patients with and without myelotoxicity (mean difference $115.54,95 \% \mathrm{CI}-109.31 ; 340.40 ; p=0.31$ ), in the pooled analysis of two studies [46,58], but with considerable heterogeneity $\left(p<0.01, \mathrm{I}^{2}=100 \%\right)$.

\section{Anemia}

\section{6-TGN}

On this topic, 5 studies $[34,36,48,81,108]$ evaluated the relation between 6-TGN levels and hemoglobin and registered a significant but weak weighted mean correlation (Figure 2a). When the analysis was restricted to studies with IBD patients $[34,36,48]$, the strength of the correlation improved $(\mathrm{r}=-0.28,95 \% \mathrm{CI}-0.50 ;-0.06 ; p=0.01)$ (Figure S4). However, in the two studies that included patients on thiopurines monotherapy, the weighted mean correlation was not statistically significant, albeit with considerable heterogeneity $(\mathrm{r}=-0.10,95 \% \mathrm{CI}-0.36 ; 0.17 ; p=0.48)\left(p<0.01, \mathrm{I}^{2}=88 \%\right)$. Regarding the relationship between 6-TGN levels and erythrocytes count $[54,81,93,94]$, the heterogeneity between studies was substantial and the correlation between variables was significant but weak (Figure 2b). The strength of the correlation improved when the analysis was restricted to studies with patients 
on thiopurines monotherapy $(\mathrm{r}=-0.39,95 \% \mathrm{CI}-0.42 ;-0.35 ; p<0.01)$, with no heterogeneity among studies $\left(p=0.01, \mathrm{I}^{2}=0 \%\right)$.

\section{Correlation meta-analysis plot}

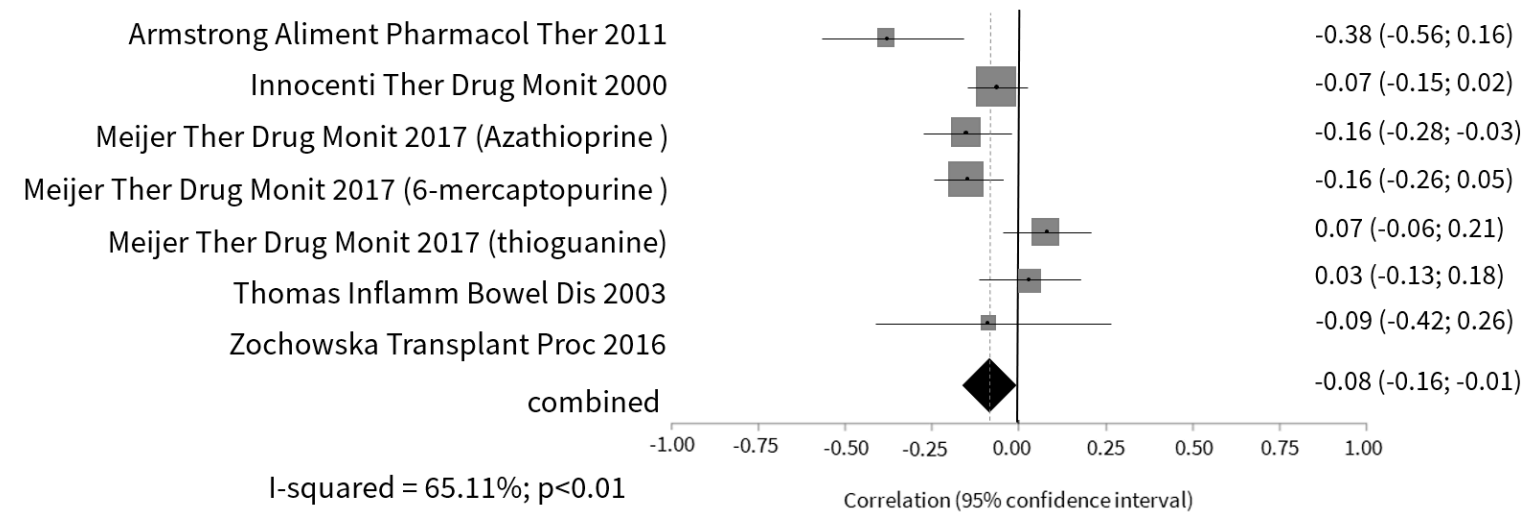

(a)

\section{Correlation meta-analysis plot}

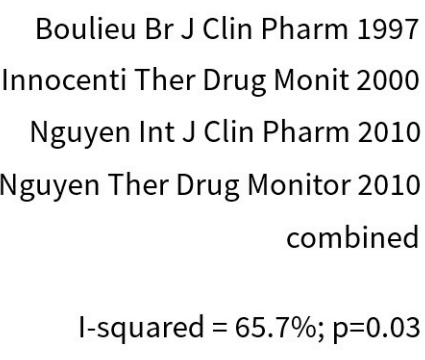

Boulieu Br J Clin Pharm 1997

nnocenti Ther Drug Monit 2000

Nguyen Int J Clin Pharm 2010

Nguyen Ther Drug Monitor 2010

I-squared $=65.7 \% ; p=0.03$

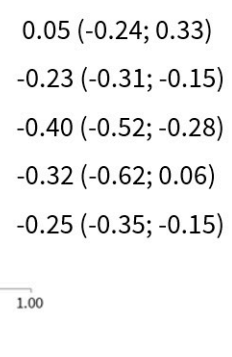

(b)

Figure 2. Correlation meta-analysis plot of 6-thioguanine nucleotides (6-TGN) and anemia. (a) Relationship between 6-TGN and hemoglobin; (b) relationship between 6-TGN and erythrocytes count.

Leukopenia

6-TGN

In a pooled analysis of 22 reports [40,46,48,56-58,65,67,70,72,73,75,84,85,91,92,94,96,98,103,105, 107], 6-TGN concentrations were significantly higher in patients with leukopenia (mean difference of $\left.127.06 \mathrm{pmol} / 8 \times 10^{8} \mathrm{RBC}(95 \% \mathrm{CI} 70.88 ; 183.24 ; p<0.01)\right)$. We could observe a considerable heterogeneity between studies $\left(p<0.01 ; \mathrm{I}^{2}=90 \%\right)$. The underlying disease showed to have influence on the results: 6-TGN levels were higher in patients with leukopenia only in leukemic and IBD patients, whereas no differences could be reported for other disorders (Figure 3a). Restricting the analysis to patients using thiopurines in monotherapy did not change the results (mean difference of $120.44 \mathrm{pmol} / 8$ $\times 10^{8} \operatorname{RBC}(95 \%$ CI 31.06; 209.83; $p<0.01)\left(p<0.01 ; \mathrm{I}^{2}=86 \%\right)$.

Twenty-six studies showed a negative correlation between 6-TGN and leukocytes, with a weak weighted mean (Figure 3b) [34,36,43,47-49,51,54,55,58,59,61,62,64,66,73,77,81,87,91,94,96,97,99,101,108]. Similar results were obtained when the analysis was limited to patients with thiopurines in monotherapy $(\mathrm{r}=-0.18,95 \% \mathrm{CI}-0.25 ;-0.12 ; p<0.01)\left(p=0.30 ; \mathrm{I}^{2}=15 \%\right)$. The strength of correlation was stronger 
when the analysis was restricted to studies with ALL patients $(\mathrm{r}=-0.35,95 \% \mathrm{CI}-0.46 ;-0.24$; $p<0.01)[58,66,73,81,101]$, pediatric cohorts $(r=-0.33,95 \% \mathrm{CI}-0.41 ;-0.25 ; p<0.01)$, and patients with $<8$ weeks of treatment at the time of the first metabolite assessment $(r=-0.31,95 \%$ CI -0.43 ; $-0.20 ; p<0.01$ ). However, a substantial heterogeneity between studies was noticed.

Nine studies provided data to calculate a pooled OR $[40,46,58,65,72,73,85,96,105]$, with thresholds ranging from 127 to $450 \mathrm{pmol} / 8 \times 10^{8} \mathrm{RBC}$. In these studies, patients with high 6-TGN levels were more than four times more likely to have leukopenia than those with lower levels (Figure 3c). In the pooled analysis of the three studies with thiopurines in monotherapy, patients with high 6-TGN levels were almost six times more likely to have leukopenia than those with lower levels (OR 5.87, 95\% CI $3.27 ; 10.55 ; p<0.01)\left(p=0.58 ; \mathrm{I}^{2}=0 \%\right)$.

Noticeably, in the sub-analysis by leukopenia definition, only studies defining leukopenia as a white blood count below 3 or $3.5 \times 109 / \mathrm{L}$ had a significant association both in mean difference (MD) and OR analysis.

The data provided for different cut-offs enabled us to calculate an optimal cut-off of $135 \mathrm{pmol} / 8$ $\times 10^{8} \mathrm{RBC}$ for leukopenia, with a sensitivity of $75.4 \%$ and specificity of $46.4 \%$ (area under the curve $($ AUC $)=0.629,95 \%$ CI 0.432; 0.837) (Table S2).

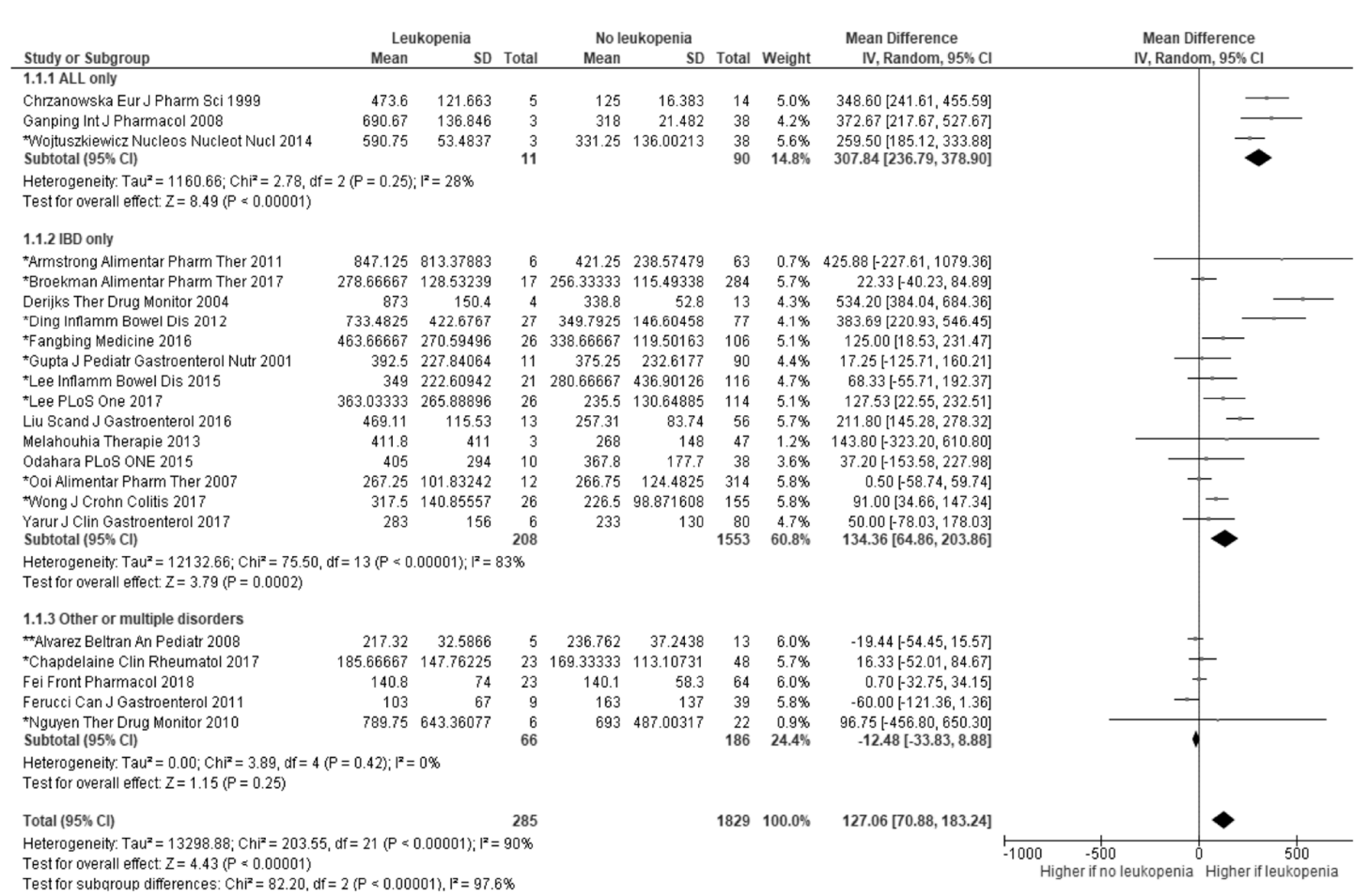

(a)

Figure 3. Cont. 
Andoh J Gastroenterol Hepatol 2008

Armstrong Aliment Pharmacol Ther 2011 Ban Gastroenterol 2010

Belaiche Scand J Gastroenterol 2001

Boulieu Adv Exp Med Biol 2000

Boulieu Br J Clin Pharm 1997

Chrzanowska Eur J Pharm Sci 1999

Chrzanowska Ther Drug Monit 1999 (renal transplant) Chrzanowska Ther Drug Monit 1999 (glomerulonephritis)

Cuffari Dig Dis Sci 2004

Dassopoulos Aliment Pharmacol Ther 2014

Derijks Eur J Gastroenterol Hepatol 2003

DervieuxLeukemia 200

Ganping Int J Pharmacol 2008

Hande Inflamm Bowel Dis 2006

Innocenti Ther Drug Monit 2000

Lennard Br J Clin Pharm 1984

Liu Scan J Gastroenterol 2016

Meijer Ther Drug Monit2017 (Azathioprine)

Meijer Ther Drug Monit2017 (6-mercaptopurine)

Meijer Ther Drug Monit2017 (6-thioguanine)

Nguyen Ther Drug Monitor 2010

Odahara PLoS One 2015

Ohtsuka J Gastroenterol Hepatol 2010

Pranzatelli J Clin Exp Immunol 2017

Sandborn Gastroenterology 1999

Schmiegelow Cancer Chemother Pharmacol 1990

Thomas Inflamm Bowel Dis 2003

Zochowska TransplantProc 2016

combined

1-squared $=72.3 \% ; p<0.01$

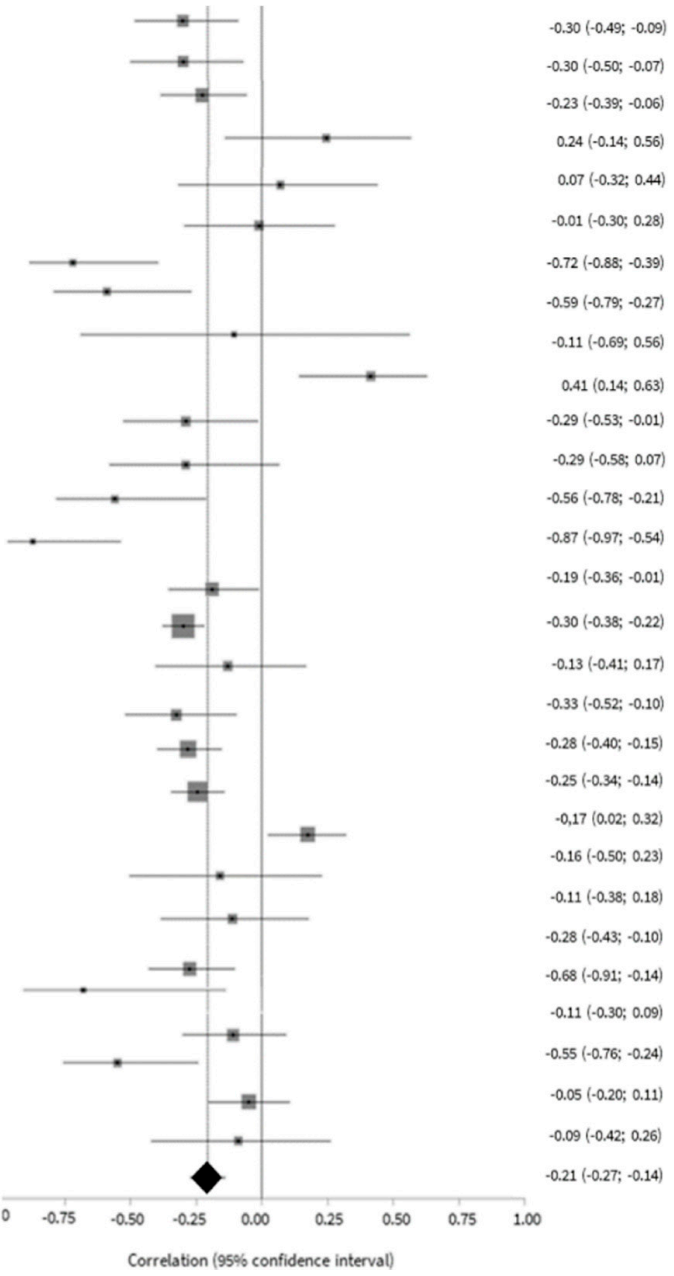

(b)

\section{Pooled Odds Ratio meta-analysis plot}

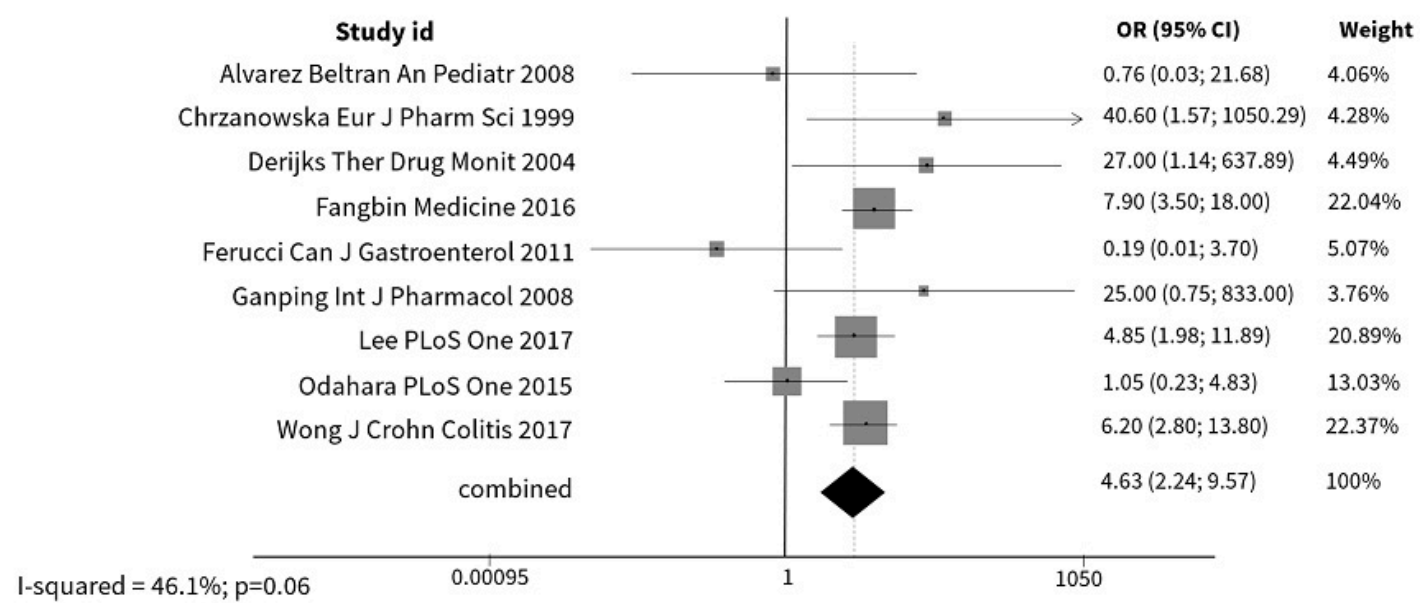

(c)

Figure 3. Relationship between 6-TGN levels and leukopenia. (a) Means difference forest plot with sub-analysis by disorder $\left({ }^{*}\right.$ studies in which mean was calculated from medians; ${ }^{* *}$ studies in which mean was calculated from a sample with skewed distribution; ALL: acute lymphoid leukemia; IBD: inflammatory bowel disease); (b) correlation meta-analysis plot; (c) odds ratio meta-analysis plot. 


\section{6-MMPR}

The analysis of a pool of six studies demonstrated that the levels of 6-MMPR were not significantly higher in patients with leukopenia $[46,58,72,85,92,105]$ (mean difference of $277.00 \mathrm{pmol} / 8 \times 10^{8} \mathrm{RBC}$ (95\% CI -237.06; 791.07; $p=0.29$ ), with similar results when the analysis was limited to patients medicated with thiopurines in monotherapy (mean difference of $1161.78 \mathrm{pmol} / 8 \times 10^{8} \mathrm{RBC}(95 \% \mathrm{CI}$ -934.44; 3258.01; $p=0.28$ ) The heterogeneity between studies was substantial $\left(p<0.01\right.$ for both; $\mathrm{I}^{2}=67 \%$ and $84 \%$, respectively). In the subgroup analysis, method and time of treatment were significant variables. In fact, only studies using the Lennard method for metabolites assessment, and studies including patients with less than 8 weeks of treatment at the time of measurement reported higher levels of 6-MMPR with leukopenia (Figure S5).

Our analysis could not denote a significant correlation between 6-MMPR levels and leucocytes $(\mathrm{r}=-0.04,95 \%$ CI $-0.12 ; 0.04 ; p=0.29)$ (Figure S6) $[36,37,55,58,94,108]$. However, when the analysis was restricted to studies including patients with less than 8 weeks of treatment at the time of assessment, the correlation was significant $(r=-0.22,95 \% \mathrm{CI}-0.34 ;-0.09 ; p<0.01)$.

The pooled analysis of three studies that provided data for OR calculation revealed that there was no association between 6-MMPR levels and leukopenia (OR 2.02, 95\% CI 0.37; 10.90; $p=0.42)(p=0.09$; $\mathrm{I}^{2}=59 \%$ ). However, in the one study that only included patients with less than 8 weeks of treatment at the time of assessment [105], patients with 6-MMPR levels above $3525 \mathrm{pmol} / 8 \times 10^{8} \mathrm{RBC}$ were almost six times more likely to develop leukopenia (OR 5.9, 95\% CI 2.7-13.3) [46,58,105]. Additionally, of the three studies, this was the only study reporting the use of thiopurines in monotherapy.

\section{6-MMPR/6-TGN Ratio}

We could evidence that the 6-MMPR/6-TGN ratio was not significantly different in patients with leukopenia [46,56,58,105] (Figure S7). Two studies correlated this ratio with leukocytes [55,58], with a weighted mean correlation of $0.31(95 \%$ CI $0.15 ; 0.46, p<0.01)\left(p=0.44 ; \mathrm{I}^{2}=0 \%\right)$.

\section{Neutropenia}

\section{6-TGN}

The mean levels of 6-TGN were similar in neutropenic and non-neutropenic patients, but there was a considerable degree of heterogeneity (MD $249.01 \mathrm{pmol} / 8 \times 10^{8} \mathrm{RBC} ; 95 \% \mathrm{CI}-276.97 ; 774.99$; $p=0.35)\left(p<0.01 ; \mathrm{I}^{2}=98 \%\right)[58,84]$. Two studies calculated the mean level of neutrophils above and below a threshold of 200 and $210 \mathrm{pmol} / 8 \times 10^{8} \mathrm{RBC}$ of 6-TGN [52,86]. The levels of neutrophils were significantly higher in the low 6-TGN group (Figure S8).

A significant negative correlation between 6-TGN and neutrophils was registered in the pooled analysis of 10 studies $[36,48,51,58,81,83,86-88,90]$ (Figure 4). This correlation was stronger in studies with conventional thiopurines $(\mathrm{r}=-0.27,95 \% \mathrm{CI}-0.36 ;-0.19 ; p<0.01)$ than in those with 6 -thioguanine, in which the correlation was in the inverse direction $(\mathrm{r}=0.18,95 \%$ CI $0.01 ; 0.34 ; p=0.04)$. However, heterogeneity was substantial. Treatment duration was shown to contribute to a stronger correlation. In fact, the weighted correlation was greater in studies including patients with less and more than 8 weeks of treatment $\left(-0.41(95 \% \mathrm{CI}-0.78 ;-0.04 ; p=0.03)\left(p=0.03 ; \mathrm{I}^{2}=78 \%\right)\right.$ versus $-0.26(95 \% \mathrm{CI}-0.37 ;-0.15$; $\left.p<0.01)\left(p<0.01 ; \mathrm{I}^{2}=72 \%\right)\right)$. When the analysis was restricted to the three studies reporting using thiopurines in monotherapy, the correlation was not significant $(\mathrm{r}=-0.12,95 \% \mathrm{CI}-0.29 ; 0.05 ; p=0.15)$ $\left(p=0.22 ; \mathrm{I}^{2}=33 \%\right)$, but all these studies included patients with more than 3 months of treatment. 


\section{Correlation meta-analysis plot}

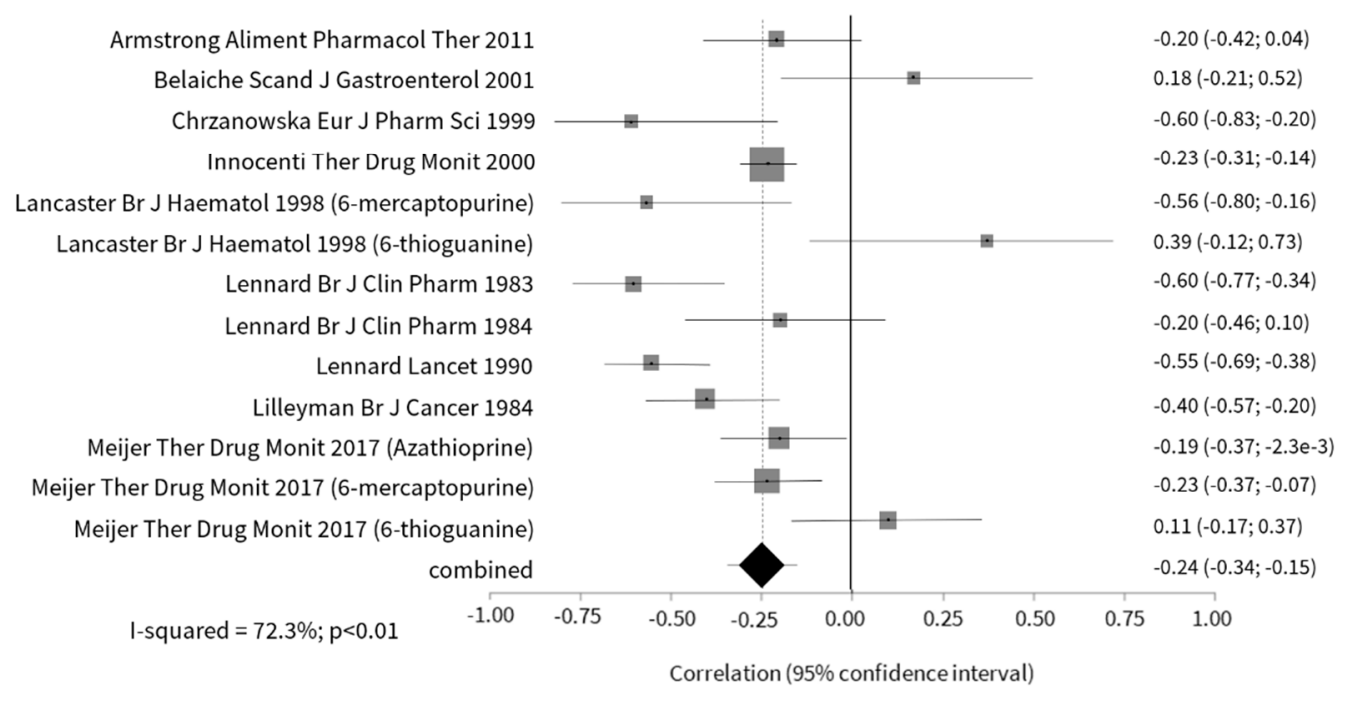

Figure 4. Correlation meta-analysis plot of 6-TGN levels and neutrophils.

Lymphopenia

In the pooled analysis of studies evaluating 6-TGN levels $[51,54,78,94,100]$ and those evaluating 6-MMPR levels $[94,100]$, no significant correlation was found between metabolites and lymphocytes ( $\mathrm{r}$ $=-0.02$ and $\mathrm{r}=-0.18$, respectively; $p>0.05$ for both). However, when we limited the analysis to studies reporting the use of thiopurines in monotherapy, there was a weak but significant correlation between 6-TGN levels and lymphocytes $(\mathrm{r}=-0.15 ; 95 \% \mathrm{CI}-0.26 ;-0.04 ; p<0.01)\left(p=0.73 ; \mathrm{I}^{2}=0 \%\right)$. The two studies evaluating correlation with 6-MMPR were also with patients on thiopurines monotherapy.

Thrombocytopenia

6-TGN

In the pooled analysis of five studies $[34,36,48,81,108]$, no significant correlation was found between 6-TGN and platelets (Figure S9). However, when patients treated with 6-thioguanine were excluded, a weak but significant correlation between these variables was observed, with less heterogeneity between studies $(\mathrm{r}=0.10,95 \% \mathrm{CI}-0.18 ;-0.02 ; p=0.02)\left(p=0.01 ; \mathrm{I}^{2}=64 \%\right)$. Similar results were obtained when the analysis was restricted to those studies reporting the use of thiopurines in monotherapy $(\mathrm{r}=-0.14$; $95 \%$ CI $-0.21 ;-0.06 ; p<0.01)\left(p=0.40 ; \mathrm{I}^{2}=0 \%\right)$.

\subsubsection{Liver Toxicity}

\section{Altered Liver Enzymes}

\section{6-TGN}

In the two included studies evaluating this relationship, levels of 6-TGN were significantly lower in patients with liver toxicity (mean difference of $-40.6 \times 10^{8} \mathrm{RBC}, 95 \% \mathrm{CI}-69.99 ;-11.22$; $p<0.01)\left(p=0.67 ; \mathrm{I}^{2}=0 \%\right)[53,104]$. We could also notice a significant negative correlation between 6-TGN levels and alanine aminotransferase (ALT), with a weighted mean correlation of -0.24 (95\% CI $-0.37 ;-0.11 ; p<0.01)\left(p=0.52 ; \mathrm{I}^{2}=0 \%\right)[76,95,108]$. Of these studies, only one used thiopurines in monotherapy [104]. 


\section{6-MMPR}

Our analysis evidenced that 6-MMPR concentrations were higher in patients with hepatotoxicity, with a mean difference of $3241.2 \mathrm{pmol} / 8 \times 10^{8} \mathrm{RBC}$ (Figure 5a) $[44,53,68,104,106]$. Nonetheless, the degree of heterogeneity between these studies was considerable. Restricting the analysis to studies reporting the use of thiopurines in monotherapy lead to non-statistically significant differences, also with considerable heterogeneity (mean difference of $5021.01 \mathrm{pmol} \times 10^{8} \mathrm{RBC}, 95 \% \mathrm{CI}-5987.12$; $16,029.15 ; p=0.37)\left(p<0.01 ; \mathrm{I}^{2}=91 \%\right)$.

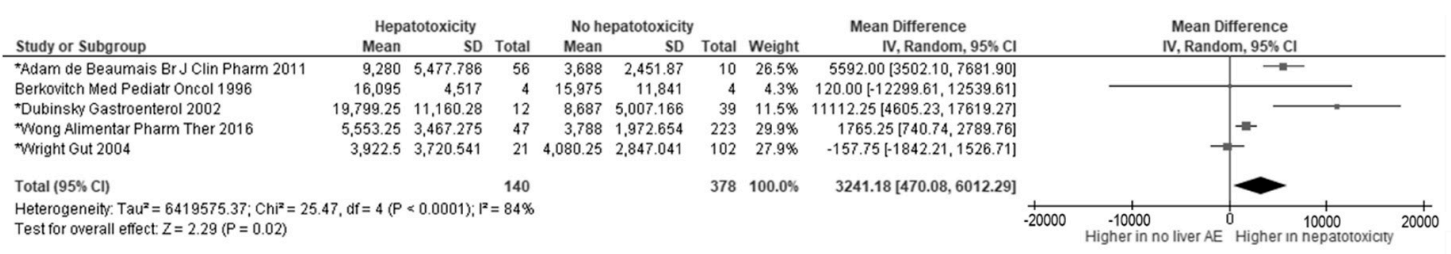

(a)

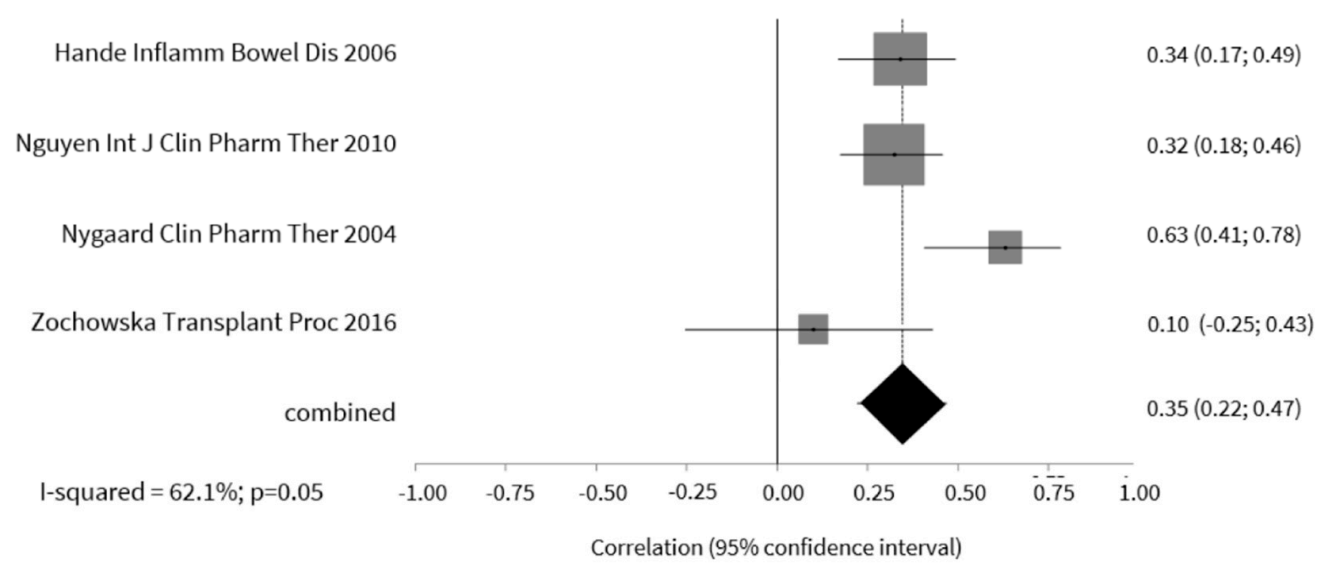

(b)

\section{Pooled Odds Ratio meta-analysis plot}

\section{Study id}

Adam de Beaumais Br J Clin Pharm 2011 Banerjee J Pediatr Gastroenterol Nutr 2006

Chapdelaine J Clin Rheum 2017

Dubinsky Gastroenterology 2000

Gupta J Pediatr Gastroenterol Nutr 2001

Kopylov J Pediatr Gastroenterol Nutr 2014

Meijer Ther Drug Monitor 2017 (conventional thiopurines)

Shaye Am J Gastroenterol 2007

Wong Aliment Pharmacol Ther 2016

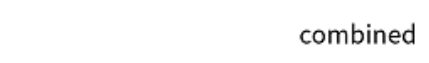

0.0113

combined

I-squared $=0.0 \% ; p=0.82$
OR $(95 \% \mathrm{Cl}) \quad$ Weight

$16.36(3.04 ; 88.15) \quad 2.96 \%$

$3.10(0.78 ; 12.24) \quad 4.44 \%$

$4.36(1.18 ; 16.13) \quad 4.90 \%$

$3.49(1.22 ; 9.96) \quad 7.61 \%$

$3.10(0.48 ; 19.99) \quad 2.41 \%$

$5.03(3.16 ; 7.98) \quad 39.09 \%$

$3.19(1.66 ; 6.12) \quad 19.65 \%$

$5.38(1.23 ; 23.55) \quad 3.85 \%$

$3.80(1.80 ; 8.00) \quad 15.08 \%$

$4.28(3.20 ; 5.71) \quad 100 \%$

(c)

Figure 5. Relationship between 6-MMPR levels and hepatotoxicity. (a) Means difference meta-analysis plot ( ${ }^{*}$ studies in which mean was calculated from medians); (b) correlation meta-analysis plot; (c) odds ratio meta-analysis plot. 
Regarding the relationship of 6-MMPR with ALT, we could observe a significant positive correlation, but with substantial heterogeneity, in four studies (Figure $5 b)[77,93,95,108]$. The results were similar when the analysis was restricted to studies with thiopurines monotherapy $(\mathrm{r}=0.33 ; 95 \% \mathrm{CI} 0.32 ; 0.34$; $p<0.01)\left(p=0.88 ; \mathrm{I}^{2}=0 \%\right)$.

Nine studies provided data for the calculation of a pooled OR $[14,36,44,50,57,75,82,102,104]$. It was possible to conclude that patients with 6-MMPR levels above the defined thresholds, ranging from 3615 to $5700 \mathrm{pmol} / 8 \times 10^{8} \mathrm{RBC}$, were about four times more likely to develop hepatotoxicity (Figure 5c). When the analysis was restricted to studies reporting the use of thiopurines in monotherapy, the results were similar (OR 4.78; 95\% CI 3.18; 7.19; $p<0.01)\left(p=0.42 ; \mathrm{I}^{2}=0 \%\right.$ ).

\section{6-MMPR/6-TGN Ratio}

Our analysis showed that there was no significant association between 6-MMPR/6-TGN ratio (thresholds of 20 [57] and 24 [82]) and liver toxicity (OR 2.9, 95\% CI 0.74; 11.55; $p=0.13)(p=0.03$; $\left.\mathrm{I}^{2}=80 \%\right)$.

\section{Veno-occlusive Disease}

In the pooled analysis of the two studies that evaluated the relationship between 6-TGN levels and the occurrence of veno-occlusive disease (VOD) $[42,89]$, no difference was found in the 6-TGN levels in patients with this type of toxicity (mean difference $7.95 \mathrm{pmol} / 8 \times 10^{8} \mathrm{RBC}, 95 \% \mathrm{CI}-118.57$; $134.47 ; p=0.90)\left(p=0.29 ; \mathrm{I}^{2}=10 \%\right)$.

\subsubsection{Gastrointestinal Intolerance}

\section{6-TGN}

The pooled analysis of two studies revealed that patients with gastrointestinal intolerance presented higher levels of 6-TGN, with a mean difference of $201.46 \mathrm{pmol} / 8 \times 10^{8} \mathrm{RBC}(95 \% \mathrm{CI} 16.86$; $386.06 ; p=0.03)\left(p=0.23 ; \mathrm{I}^{2}=29 \%\right)[67,79]$.

\subsubsection{Pancreatitis, Infections and NRH}

For each of these adverse events, only one study provided data on metabolites [66,80,109]. As such, it was not possible to perform meta-analysis, and these studies were not included.

\section{Discussion}

The wide use of thiopurines has been hindered by their inherent toxicity, which may also result in underdosing and lack of efficacy [5]. The measurement of thiopurines' metabolites can give physicians a safer context for prescription, if the levels are kept in the therapeutic range and below toxic thresholds. However, the therapeutic range may differ depending on the disorder and on the indication. In IBD, the most cited optimal range is of $\sim 230$ to $400 \mathrm{pmol} / 8 \times 10^{8} \mathrm{RBC}$, when thiopurines are used in monotherapy $[12,13]$. Still, thiopurines are also important in combination with anti-TNF drugs, and may be the most important factor in reducing the immunogenicity of these drugs [110,111]. In combination therapy with biologics, lower levels (105 to $125 \mathrm{pmol} / 8 \times 10^{8} \mathrm{RBC}$ ) may be enough to improve anti-TNF pharmacokinetics [112,113].

In this study, we identified a relationship between thiopurines' metabolites and several adverse events: (i) 6-TGN were associated with leukopenia, neutropenia and gastrointestinal intolerance, and inversely associated with liver toxicity; and (ii) 6-MMPR were associated with liver toxicity and early leukopenia. As myelosuppression has long been linked to 6-TGN, we calculated an optimal 6-TGN threshold $\left(135 \mathrm{pmol} / 8 \times 10^{8}\right)$ for the occurrence of leukopenia. Although this cut-off is below the therapeutic levels for monotherapy with thiopurines in IBD, it is above the optimal cut-off for the levels demanded in combination therapy. This constitutes an additional argument for using lower doses of thiopurines when the drug is combined with infliximab. Regarding neutrophils and 
platelets, correlation with 6-TGN was only significant when the analysis was restricted to conventional thiopurines. Scientific evidence indicates that 6-TGN levels derived from 6-thioguanine have a different impact than those resulting from conventional thiopurines. Indeed, low doses of 6-thioguanine can lead to high 6-TGN levels without evidence of myelosuppression [114]. Some explanations can be pointed out for this fact. As most methods do not measure 6-TGN directly, but reduce it to thioguanine, the ingested 6-thioguanine is indistinguishable from 6-TGN, resulting in false high levels of 6-TGN if the drug is ingested close to the assay [115]. In addition, 6-MMPR are not produced with 6-thioguanine. Our results evidenced a possible association of early 6-MMPR assessment (i.e., in the first 8 weeks of treatment) with leukopenia. In previous studies, these metabolites were shown to be cytotoxic and to inhibit purine de novo synthesis, contributing to the antiproliferative properties of these drugs, responsible for both therapeutic and myelotoxic effects [116].

We have also confirmed a positive association of hepatoxicity with 6-MMPR, and a negative association with 6-TGN. In patients who metabolize thiopurines preferentially through the methylation pathway, generating high levels of 6-MMPR [13] (known as "shunters"), dose escalation will not always improve clinical outcomes. This explains thiopurines' inefficacy despite optimal weight-based dosage [68]. Some strategies can be used to improve the metabolite profile in these patients: (i) dose-splitting regimen [117]; (ii) addition of allopurinol [1,117]; or (iii) use of 6-thioguanine instead of a conventional thiopurine [114].

Gastrointestinal intolerance to thiopurines is one of the most frequent adverse events with thiopurines treatment, causing many patients to abandon treatment [118]. Some authors postulated that this adverse event could be related to the nitro-imidazole compound released in AZA metabolism to form 6-MP [119]. In this way, 6-MP could be an adequate alternative to AZA treatment in patients experiencing gastrointestinal intolerance, as was demonstrated in some studies [119,120]. However, we have found that 6-TGN levels were associated with the occurrence of gastrointestinal intolerance. Accordingly, in one of the studies included in the analysis, switch of AZA to 6-MP was only tolerated in a small proportion of patients [79].

The reported higher sensitivity of Asian populations to thiopurines, when compared to Western populations, deserves particular consideration [121]. In this context and whenever possible, we performed subgroup-analysis according to the geographical origin of the studies. Generally, we could not evidence a significant influence of ethnicity on toxicity. Differences in metabolism are probably responsible for these "different sensitivities". These results are strong arguments in favor of metabolites' monitoring rather than the traditional weight-based strategy. In fact, even though metabolism may vary according to ethnicity, the significance of concentrations of metabolites is likely similar across populations.

The results of this meta-analysis were impacted by several limitations. Most studies were retrospective, with small samples and, in many of them, the evaluation of the relationship between metabolites and toxicity was a secondary outcome. As such, toxicity events were possibly not always registered, and samples were often underpowered for conclusive results. The mean global quality score was only $58.9 \%$, reflecting these factors. In most cases, studies displayed a high degree of heterogeneity and the definitions of toxic events were inconsistent, suggesting that the results should be interpreted with caution. This heterogeneity was, in part, caused by the different methods applied for the measurement of metabolites. Even though conversion factors for 6-TGN have been described to uniformize values, small variations in protocols can lead to significant differences in 6-TGN concentrations [1,31,35]. Levels of 6-MMPR are reported as being similar in different assays [35-37], but this causes a problem in the definition of optimal values for 6-MMPR/6-TGN ratios. Cut-offs obtained by a specific assay are not interchangeable [31] and thus the calculated optimal threshold for leukopenia should be used with caution in clinical practice. In the future, the standardization of procedures for the assessment of metabolites is of upmost importance [41,117]. Another limitation of this analysis is that most studies included patients with more than 8 weeks of treatment, but most adverse events with thiopurines occur in the first weeks/months $[4,5,79]$. Hence, a large proportion of patients had 
their doses of drug reduced (or even discontinued) at the time of the study. The strength of association between metabolites and adverse events could be stronger if more studies included patients in the beginning of thiopurine treatment. In fact, Wong et al. demonstrated that the assessment of metabolites at week 1 could predict the later occurrence of leukopenia and hepatotoxicity [104,105]. Some authors reduce the doses of thiopurines or change the treatment strategy in patients with high 6-MMPR based on similar experiences in their clinical practice, but this strategy is yet to be validated. [68] To perform this meta-analysis, we converted median values (reported in most studies) to mean values. This procedure could have introduced some error in the final analysis. However, whenever possible, we performed sub-analysis by "provided vs. calculated means", with no impact on the results. To finalize, many studies were excluded from the meta-analysis for not providing enough data for calculations. Most of these studies mentioned the absence of significant relationships between metabolites and adverse events, which might result in a positive results bias.

As many thiopurine-associated adverse events are related to the level of metabolites, physicians should take this information into account for dose selection, to achieve the best compromise between efficacy and toxicity. The importance of establishing a clear relationship between metabolite levels and toxicity may also be of value in patients receiving concomitant medications with similar toxicity profiles. In these cases, metabolites' measurement will help to determine the culprit. The same applies to disorders in which the clinical presentation resembles drug toxicity, as in the case of AIH flares. If the context enables TPMT and nudix hydrolase-15 (NUDT15) screening before starting the treatment to inform on eligibility or drug dosage, subsequent adjustments can be guided by the measurement of metabolites, in a tiered approach [117]. However, at this point, these strategies should be used as adjuncts in clinical practice and cannot yet replace blood and clinical monitoring for early detection of toxicity. A more personalized medicine should overcome the traditional weight-based dosing of thiopurines and rely more on TDM. Still, higher quality studies are needed to confirm this strategy.

Supplementary Materials: The following are available online at http://www.mdpi.com/2077-0383/9/7/2216/s1, Figure S1: Relationship between 6-TGN levels and overall toxicity: mean difference forest plot (AE-adverse events) with sub-analysis by age group; Figure S2: Relationship between 6-MMPR levels and overall toxicity: mean difference forest plot (AE-adverse events); Figure S3: Relationship between 6-TGN levels and overall myelotoxicity: mean difference forest plot with sub-analysis by duration of treatment; Figure S4: Relationship between 6-TGN levels and hemoglobin: correlation meta-analysis plot and sub-analysis of studies including patients with inflammatory bowel disease; Figure S5: Mean difference forest plot of 6-MMPR levels and leukopenia: (a) sub-analysis by method (b) sub-analysis by treatment duration; Figure S6: Relationship between 6 MMPR levels and leukopenia: correlation meta-analysis plot; Figure S7: Relationship between 6-MMPR/6-TGN ratio and leukopenia: mean difference forest plot; Figure S8: Relationship between neutrophils levels and 6-TGN levels: mean difference forest plot; Figure S9: Correlation meta-analysis plot of 6-TGN and platelets; Table S1: Type and definition of toxicities evaluated in each study included in the meta-analysis; Table S2: Measures of performance of different 6-TGN cut-offs for leukopenia occurrence (calculated with the method described in Steinhauser S, Schumacher M, Rucker G. Modelling multiple thresholds in meta-analysis of diagnostic test accuracy studies. BMC medical research methodology. 2016;16(1):97).

Author Contributions: Conceptualization, P.S. and F.M.; methodology, P.S., M.M.E., C.C.D. and F.M.; validation, M.M.E. and C.C.D.; formal analysis, P.S., M.M.E. and C.C.D.; investigation, P.S., M.M.E., C.C.D. and F.M.; resources, P.S., M.M.E., C.C.D., P.M. and F.M.; data curation, P.S., M.M.E. and C.C.D.; writing-original draft preparation, P.S.; writing-review and editing, M.M.E., C.C.D., P.M., U.K., S.D., L.P.-B., F.M.; visualization, P.S., M.M.E., C.C.D., F.M.; supervision, P.M., U.K., S.D., L.P.-B., F.M.; project administration, F.M.; funding acquisition, P.S., P.M., F.M. All authors have read and agreed to the published version of the manuscript.

Funding: This research was supported by an Doctoral Scholarship from the Portuguese Group for the Study of Inflammatory Bowel Disease (Grupo de Estudos de Doença Inflamatória Intestinal—GEDII) to P.S. in 2018.

Acknowledgments: The authors thank Paula Pinto, (PMA-Pharmaceutical Medicine Academy) for providing medical writing and editorial assistance.

Conflicts of Interest: P.M. is a consultant for Abbvie, Ferring, Hospira, Janssen, MSD, Pfizer, Takeda. U.K. has received speaker fees from Abbvie, Janssen, Medtronic, and Takeda, research support from Takeda, Medtronic and Janssen, and consulting fees from Takeda, Medtronic and Abbvie. S.D. has served as a speaker, a consultant and an advisory board member for Abbvie, Ferring, Hospira, Johnson \& Johnson, Merck, Millennium Takeda, Mundipharma, Pfizer, Tigenix, UCB Pharma and Vifor. L.P.-B. received honoraria from Merck, Abbvie, Janssen, Genentech, Mitsubishi, Ferring, Norgine, Tillots, Vifor, Hospira/Pfizer, Celltrion, Takeda, Biogaran, Boerhinger-Ingelheim, Lilly, HAC-Pharma, Index Pharmaceuticals, Amgen, Sandoz, Forward Pharma GmbH, 
Celgene, Biogen, Lycera, Samsung Bioepis. F.M. has served as a speaker and received honoraria from Merck Sharp \& Dohme, Abbvie, Vifor, Falk, Laboratorios Vitoria, Ferring, Hospira, and Biogen. The other authors have no conflicts of interest to disclose. The funders had no role in the design of the study; in the collection, analyses, or interpretation of data; in the writing of the manuscript, or in the decision to publish the results.

\section{References}

1. Van Asseldonk, D.P.; de Boer, N.K.; Peters, G.J.; Veldkamp, A.I.; Mulder, C.J.; Van Bodegraven, A.A. On therapeutic drug monitoring of thiopurines in inflammatory bowel disease; pharmacology, pharmacogenomics, drug intolerance and clinical relevance. Curr. Drug Metab. 2009, 10, 981-997. [CrossRef]

2. Lennard, L.; Singleton, H.J. High-performance liquid chromatographic assay of the methyl and nucleotide metabolites of 6-mercaptopurine: Quantitation of red blood cell 6-thioguanine nucleotide, 6-thioinosinic acid and 6-methylmercaptopurine metabolites in a single sample. J. Chromatogr. 1992, 583, 83-90. [CrossRef]

3. Dervieux, T.; Boulieu, R. Simultaneous determination of 6-thioguanine and methyl 6-mercaptopurine nucleotides of azathioprine in red blood cells by HPLC. Clin. Chem. 1998, 44, 551-555. [CrossRef]

4. Gisbert, J.P.; Gomollon, F. Thiopurine-induced myelotoxicity in patients with inflammatory bowel disease: A review. Am. J. Gastroenterol. 2008, 103, 1783-1800. [CrossRef] [PubMed]

5. Chaparro, M.; Ordas, I.; Cabre, E.; Garcia-Sanchez, V.; Bastida, G.; Penalva, M.; Gomollon, F.; Garcia-Planella, E.; Merino, O.; Gutierrez, A.; et al. Safety of thiopurine therapy in inflammatory bowel disease: Long-term follow-up study of 3931 patients. Inflamm. Bowel Dis. 2013, 19, 1404-1410. [CrossRef] [PubMed]

6. Van Asseldonk, D.P.; Sanderson, J.; de Boer, N.K.; Sparrow, M.P.; Lemann, M.; Ansari, A.; Almer, S.H.; Florin, T.H.; Gearry, R.B.; Mulder, C.J.; et al. Difficulties and possibilities with thiopurine therapy in inflammatory bowel disease-Proceedings of the first Thiopurine Task Force meeting. Dig. Liver Dis. 2011, 43, 270-276. [CrossRef]

7. Beswick, L.; Friedman, A.B.; Sparrow, M.P. The role of thiopurine metabolite monitoring in inflammatory bowel disease. Expert Rev. Gastroenterol. 2014, 8, 383-392. [CrossRef]

8. Lilleyman, J.S.; Lennard, L. Mercaptopurine metabolism and risk of relapse in childhood lymphoblastic leukaemia. Lancet 1994, 343, 1188-1190. [CrossRef]

9. Lennard, L.; Lilleyman, J.S. Are children with lymphoblastic leukaemia given enough 6-mercaptopurine? Lancet 1987, 330, 785-787. [CrossRef]

10. Estevinho, M.M.; Afonso, J.; Rosa, I.; Lago, P.; Trindade, E.; Correia, L.; Dias, C.C.; Magro, F. A Systematic Review and Meta-Analysis of 6-Thioguanine Nucleotide Levels and Clinical Remission in Inflammatory Bowel Disease. J. Crohns Colitis 2017, 11, 1381-1392. [CrossRef]

11. Bergan, S.; Rugstad, H.E.; Bentdal, O.; Sodal, G.; Hartmann, A.; Leivestad, T.; Stokke, O. Monitored high-dose azathioprine treatment reduces acute rejection episodes after renal transplantation. Transplantation 1998, 66, 334-339. [CrossRef] [PubMed]

12. Osterman, M.T.; Kundu, R.; Lichtenstein, G.R.; Lewis, J.D. Association of 6-thioguanine nucleotide levels and inflammatory bowel disease activity: A meta-analysis. Gastroenterology 2006, 130, 1047-1053. [CrossRef] [PubMed]

13. Haglund, S. Interindividual Differences in Thiopurine Metabolism: Studies with Focus on Inflammatory Bowel Disease. Ph.D. Thesis, Linköping University, Linköping, Sweden, 2011.

14. Dubinsky, M.C.; Lamothe, S.; Yang, H.Y.; Targan, S.R.; Sinnett, D.; Theoret, Y.; Seidman, E.G. Pharmacogenomics and metabolite measurement for 6-mercaptopurine therapy in inflammatory bowel disease. Gastroenterology 2000, 118, 705-713. [CrossRef]

15. Gearry, R.B.; Barclay, M.L. Azathioprine and 6-mercaptopurine pharmacogenetics and metabolite monitoring in inflammatory bowel disease. J. Gastroen. Hepatol. 2005, 20, 1149-1157. [CrossRef]

16. Vande Casteele, N.; Herfarth, H.; Katz, J.; Falck-Ytter, Y.; Singh, S. American Gastroenterological Association Institute Technical Review on the Role of Therapeutic Drug Monitoring in the Management of Inflammatory Bowel Diseases. Gastroenterology 2017, 153, 835-857. [CrossRef]

17. Azathioprine- Azathioprine Tablet [Package Insert]. Available online: https://dailymed.nlm.nih.gov/ dailymed/drugInfo.cfm?setid=ceab8e8b-d022-4d0c-a552-cc5782446248 (accessed on 5 February 2020). 
18. Relling, M.V.; Gardner, E.E.; Sandborn, W.J.; Schmiegelow, K.; Pui, C.H.; Yee, S.W.; Stein, C.M.; Carrillo, M.; Evans, W.E.; Klein, T.E.; et al. Clinical Pharmacogenetics Implementation Consortium guidelines for thiopurine methyltransferase genotype and thiopurine dosing. Clin. Pharmacol. Ther. 2011, 89, 387-391. [CrossRef]

19. Relling, M.V.; Gardner, E.E.; Sandborn, W.J.; Schmiegelow, K.; Pui, C.H.; Yee, S.W.; Stein, C.M.; Carrillo, M.; Evans, W.E.; Hicks, J.K.; et al. Clinical pharmacogenetics implementation consortium guidelines for thiopurine methyltransferase genotype and thiopurine dosing: 2013 update. Clin. Pharmacol. Ther. 2013, 93, 324-325. [CrossRef]

20. Ribaldone, D.G.; Adriani, A.; Caviglia, G.P.; Nicolò, A.; Agnesod, D.; Simiele, M.; Riganò, D.; Pellicano, R.; Canaparo, R.; Perri, G.D.; et al. Correlation between Thiopurine S-Methyltransferase Genotype and Adverse Events in Inflammatory Bowel Disease Patients. Medicina 2019, 55, 441. [CrossRef]

21. Moher, D.; Liberati, A.; Tetzlaff, J.; Altman, D.G. Preferred reporting items for systematic reviews and meta-analyses: The PRISMA statement. PLoS Med. 2009, 6, e1000097. [CrossRef]

22. Jonathan, J.; Deeks, J.P.H.; Altman, D.G. Chapter 10: Analysing data and undertaking meta-analyses. In Cochrane Handbook for Systematic Reviews of Interventions Version 6.0 (Updated July 2019); Higgins, J.P.T., Thomas, J., Chandler, J., Cumpston, M., Li, T., Page, M.J., Welch, V.A., Eds.; Cochrane: London, UK, 2019.

23. PubMed@. Available online: https://www.ncbi.nlm.nih.gov/pubmed/ (accessed on 3 November 2018).

24. Web of Science. Available online: http://www.isiwebofknowledge.com (accessed on 3 November 2018).

25. Scopus. Available online: https://www.elsevier.com/solutions/scopus (accessed on 3 November 2018).

26. Ouzzani, M.; Hammady, H.; Fedorowicz, Z.; Elmagarmid, A. Rayyan-a web and mobile app for systematic reviews. Syst. Rev. 2016, 5, 210. [CrossRef]

27. Sirriyeh, R.; Lawton, R.; Gardner, P.; Armitage, G. Reviewing studies with diverse designs: The development and evaluation of a new tool. J. Eval. Clin. Pract. 2012, 18, 746-752. [CrossRef] [PubMed]

28. Wan, X.; Wang, W.; Liu, J.; Tong, T. Estimating the sample mean and standard deviation from the sample size, median, range and/or interquartile range. BMC Med. Res. Methodol. 2014, 14, 135. [CrossRef]

29. Shipkova, M.; Armstrong, V.W.; Wieland, E.; Oellerich, M. Differences in nucleotide hydrolysis contribute to the differences between erythrocyte 6-thioguanine nucleotide concentrations determined by two widely used methods. Clin. Chem. 2003, 49, 260-268. [CrossRef] [PubMed]

30. Lennard, L. Assay of 6-thioinosinic acid and 6-thioguanine nucleotides, active metabolites of 6-mercaptopurine, in human red blood cells. J. Chromatogr. 1987, 423, 169-178. [CrossRef]

31. Armstrong, V.W.; Shipkova, M.; von Ahsen, N.; Oellerich, M. Analytic aspects of monitoring therapy with thiopurine medications. Ther. Drug Monit. 2004, 26, 220-226. [CrossRef] [PubMed]

32. Erdmann, G.R.; France, L.A.; Bostrom, B.C.; Canafax, D.M. A reversed phase high performance liquid chromatography approach in determining total red blood cell concentrations of 6-thioguanine, 6-mercaptopurine, methylthioguanine, and methylmercaptopurine in a patient receiving thiopurine therapy. Biomed. Chromatogr. 1990, 4, 47-51. [CrossRef] [PubMed]

33. Lowry, P.W.; Franklin, C.L.; Weaver, A.L.; Pike, M.G.; Mays, D.C.; Tremaine, W.J.; Lipsky, J.J.; Sandborn, W.J. Measurement of thiopurine methyltransferase activity and azathioprine metabolites in patients with inflammatory bowel disease. Gut 2001, 49, 665-670. [CrossRef]

34. Thomas, C.W., Jr.; Lowry, P.W.; Franklin, C.L.; Weaver, A.L.; Myhre, G.M.; Mays, D.C.; Tremaine, W.J.; Lipsky, J.J.; Sandborn, W.J. Erythrocyte mean corpuscular volume as a surrogate marker for 6-thioguanine nucleotide concentration monitoring in patients with inflammatory bowel disease treated with azathioprine or 6-mercaptopurine. Inflamm. Bowel Dis. 2003, 9, 237-245. [CrossRef]

35. Robijns, K.; van Luin, M.; Jansen, R.T.P.; Neef, C.; Touw, D.J. A design for external quality assessment for the analysis of thiopurine drugs: Pitfalls and opportunities. Clin. Chem. Lab. Med. 2018, 56, 1715-1721. [CrossRef]

36. Meijer, B.; Wilhelm, A.J.; Mulder, C.J.J.; Bouma, G.; Van Bodegraven, A.A.; de Boer, N.K.H. Pharmacology of Thiopurine Therapy in Inflammatory Bowel Disease and Complete Blood Cell Count Outcomes: A 5-Year Database Study. Ther. Drug Monit. 2017, 39, 399-405. [CrossRef]

37. Meijer, B.; Kreijne, J.E.; van Moorsel, S.A.W.; Derijks, L.J.J.; Bouma, G.; Mulder, C.J.J.; Wong, D.R.; van der Woude, C.J.; van Bodegraven, A.A.; de Boer, N.K.H. 6-methylmercaptopurine-induced leukocytopenia during thiopurine therapy in inflammatory bowel disease patients. J. Gastroenterol. Hepatol. 2017, 32, 1183-1190. [CrossRef] [PubMed] 
38. Field, A.P. Meta-analysis of correlation coefficients: A Monte Carlo comparison of fixed- and random-effects methods. Psychol. Methods 2001, 6, 161-180. [CrossRef] [PubMed]

39. Steinhauser, S.; Schumacher, M.; Rucker, G. Modelling multiple thresholds in meta-analysis of diagnostic test accuracy studies. BMC Med. Res. Methodol. 2016, 16, 97. [CrossRef] [PubMed]

40. Fangbin, Z.; Xiang, G.; Liang, D.; Hui, L.; Xueding, W.; Baili, C.; Huichang, B.; Yinglian, X.; Peng, C.; Lizi, Z.; et al. Prospective Evaluation of Pharmacogenomics and Metabolite Measurements upon Azathioprine Therapy in Inflammatory Bowel Disease: An Observational Study. Medicine 2016, 95, e3326. [CrossRef] [PubMed]

41. Vikingsson, S.; Carlsson, B.; Almer, S.H.; Peterson, C. Monitoring of thiopurine metabolites in patients with inflammatory bowel disease-what is actually measured? Ther. Drug Monit. 2009, 31, 345-350. [CrossRef] [PubMed]

42. Stoneham, S.; Lennard, L.; Coen, P.; Lilleyman, J.; Saha, V. Veno-occlusive disease in patients receiving thiopurines during maintenance therapy for childhood acute lymphoblastic leukaemia. Br. J. Haematol. 2003, 123, 100-102. [CrossRef]

43. Sandborn, W.J.; Tremaine, W.J.; Wolf, D.C.; Targan, S.R.; Sninsky, C.A.; Sutherland, L.R.; Hanauer, S.B.; McDonald, J.W.D.; Feagan, B.G.; Fedorak, R.N.; et al. Lack of effect of intravenous administration on time to respond to azathioprine for steroid-treated Crohn's disease. Gastroenterology 1999, 117, 527-535. [CrossRef]

44. Adam de Beaumais, T.; Fakhoury, M.; Medard, Y.; Azougagh, S.; Zhang, D.; Yakouben, K.; Jacqz-Aigrain, E. Determinants of mercaptopurine toxicity in paediatric acute lymphoblastic leukemia maintenance therapy. Br. J. Clin. Pharmacol. 2011, 71, 575-584. [CrossRef]

45. Almer, S.H.; Hjortswang, H.; Hindorf, U. 6-Thioguanine therapy in Crohn's disease-observational data in Swedish patients. Dig. Liver Dis. 2009, 41, 194-200. [CrossRef]

46. Alvarez Beltran, M.; Infante Pina, D.; Tormo Carnice, R.; Segarra Canton, O.; Redecillas Ferreiro, S. Optimising azathioprine treatment: Determination of thiopurine methyltransferase activity and thiopurine metabolites. An. Pediatr. 2009, 70, 126-131. [CrossRef]

47. Andoh, A.; Tsujikawa, T.; Ban, H.; Hashimoto, T.; Bamba, S.; Ogawa, A.; Sasaki, M.; Saito, Y.; Fujiyama, Y. Monitoring 6-thioguanine nucleotide concentrations in Japanese patients with inflammatory bowel disease. J. Gastroenterol. Hepatol. 2008, 23, 1373-1377. [CrossRef] [PubMed]

48. Armstrong, L.; Sharif, J.A.; Galloway, P.; McGrogan, P.; Bishop, J.; Russell, R.K. Evaluating the use of metabolite measurement in children receiving treatment with a thiopurine. Aliment. Pharmacol. Ther. 2011, 34, 1106-1114. [CrossRef]

49. Ban, H.; Andoh, A.; Imaeda, H.; Kobori, A.; Bamba, S.; Tsujikawa, T.; Sasaki, M.; Saito, Y.; Fujiyama, Y. The multidrug-resistance protein 4 polymorphism is a new factor accounting for thiopurine sensitivity in Japanese patients with inflammatory bowel disease. J. Gastroenterol. 2010, 45, 1014-1021. [CrossRef] [PubMed]

50. Banerjee, S.; Bishop, W.P. Evolution of thiopurine use in pediatric inflammatory bowel disease in an academic center. J. Pediatr. Gastroenterol. Nutr. 2006, 43, 324-330. [CrossRef] [PubMed]

51. Belaiche, J.; Desager, J.P.; Horsmans, Y.; Louis, E. Therapeutic drug monitoring of azathioprine and 6-mercaptopurine metabolites in Crohn disease. Scand. J. Gastroenterol. 2001, 36, 71-76. [CrossRef] [PubMed]

52. Bergan, S.; Rugstad, H.E.; Bentdal, O.; Stokke, O. Monitoring of azathioprine treatment by determination of 6-thioguanine nucleotide concentrations in erythrocytes. Transplantation 1994, 58, 803-808. [CrossRef]

53. Berkovitch, M.; Matsui, D.; Zipursky, A.; Blanchette, V.S.; Verjee, Z.; Giesbrecht, E.; Saunders, E.F.; Evans, W.E.; Koren, G. Hepatotoxicity of 6-mercaptopurine in childhood acute lymphocytic leukemia: Pharmacokinetic characteristics. Med. Pediatr. Oncol. 1996, 26, 85-89. [CrossRef]

54. Boulieu, R.; Lenoir, A.; Bertocchi, M.; Mornex, J.F. Intracellular thiopurine nucleotides and azathioprine myelotoxicity in organ transplant patients. Br. J. Clin. Pharmacol. 1997, 43, 116-118. [CrossRef]

55. Boulieu, R.; Dervieux, T.; Gallant, I.; Sauviat, M.; Bertocchi, M.; Mornex, J.F. Methylated and non methylated thiopurine nucleotide ratio (Me6-MPN/6-TGN): Usefulness in the monitoring of azathioprine therapy? Adv. Exp. Med. Biol. 2000, 486, 361-367. [CrossRef]

56. Broekman, M.; Coenen, M.J.H.; Wanten, G.J.; van Marrewijk, C.J.; Klungel, O.H.; Verbeek, A.L.M.; Hooymans, P.M.; Guchelaar, H.J.; Scheffer, H.; Derijks, L.J.J.; et al. Risk factors for thiopurine-induced myelosuppression and infections in inflammatory bowel disease patients with a normal TPMT genotype. Aliment. Pharmacol. Ther. 2017, 46, 953-963. [CrossRef] 
57. Chapdelaine, A.; Mansour, A.M.; Troyanov, Y.; Williamson, D.R.; Dore, M. Metabolite monitoring to guide thiopurine therapy in systemic autoimmune diseases. Clin. Rheumatol. 2017, 36, 1341-1348. [CrossRef] [PubMed]

58. Chrzanowska, M.; Kolecki, P.; Duczmal-Cichocka, B.; Fiet, J. Metabolites of mercaptopurine in red blood cells: A relationship between 6-thioguanine nucleotides and 6-methylmercaptopurine metabolite concentrations in children with lymphoblastic leukemia. Eur. J. Pharm. Sci. 1999, 8, 329-334. [CrossRef]

59. Chrzanowska, M.; Krzymanski, M. Determination of 6-thioguanine and 6-methylmercaptopurine metabolites in renal transplantation recipients and patients with glomerulonephritis treated with azathioprine. Ther. Drug Monit. 1999, 21, 231-237. [CrossRef] [PubMed]

60. Cuffari, C.; Théorêt, Y.; Latour, S.; Seidman, G. 6-Mercaptopurine metabolism in Crohn's disease: Correlation with efficacy and toxicity. Gut 1996, 39, 401-406. [CrossRef]

61. Cuffari, C.; Li, D.Y.; Mahoney, J.; Barnes, Y.; Bayless, T.M. Peripheral blood mononuclear cell DNA 6-thioguanine metabolite levels correlate with decreased interferon-gamma production in patients with Crohn's disease on AZA therapy. Dig. Dis. Sci. 2004, 49, 133-137. [CrossRef]

62. Dassopoulos, T.; Dubinsky, M.C.; Bentsen, J.L.; Martin, C.F.; Galanko, J.A.; Seidman, E.G.; Sandler, R.S.; Hanauer, S.B. Randomised clinical trial: Individualised vs. weight-based dosing of azathioprine in Crohn's disease. Aliment. Pharmacol. Ther. 2014, 39, 163-175. [CrossRef]

63. De Boer, N.K.; Derijks, L.J.; Gilissen, L.P.; Hommes, D.W.; Engels, L.G.; de-Boer, S.Y.; den Hartog, G.; Hooymans, P.M.; Makelburg, A.B.; Westerveld, B.D.; et al. On tolerability and safety of a maintenance treatment with 6-thioguanine in azathioprine or 6-mercaptopurine intolerant IBD patients. World J. Gastroenterol. 2005, 11, 5540-5544. [CrossRef]

64. Derijks, L.J.; de Jong, D.J.; Gilissen, L.P.; Engels, L.G.; Hooymans, P.M.; Jansen, J.B.; Mulder, C.J. 6-Thioguanine seems promising in azathioprine- or 6-mercaptopurine-intolerant inflammatory bowel disease patients: A short-term safety assessment. Eur. J. Gastroenterol. Hepatol. 2003, 15, 63-67. [CrossRef]

65. Derijks, L.J.; Gilissen, L.P.; Engels, L.G.; Bos, L.P.; Bus, P.J.; Lohman, J.J.; Curvers, W.L.; Van Deventer, S.J.; Hommes, D.W.; Hooymans, P.M. Pharmacokinetics of 6-mercaptopurine in patients with inflammatory bowel disease: Implications for therapy. Ther. Drug Monit. 2004, 26, 311-318. [CrossRef]

66. Dervieux, T.; Medard, Y.; Verpillat, P.; Guigonis, V.; Duval, M.; Lescoeur, B.; Suciu, S.; Vilmer, E.; Jacqz-Aigrain, E. Possible implication of thiopurine S-methyltransferase in occurrence of infectious episodes during maintenance therapy for childhood lymphoblastic leukemia with mercaptopurine. Leukemia 2001, 15, 1706-1712. [CrossRef]

67. Ding, L.; Zhang, F.B.; Liu, H.; Gao, X.; Bi, H.C.; Wang, X.D.; Chen, B.L.; Zhang, Y.; Zhao, L.Z.; Zhong, G.P.; et al. Hypoxanthine guanine phosphoribosyltransferase activity is related to 6-thioguanine nucleotide concentrations and thiopurine-induced leukopenia in the treatment of inflammatory bowel disease. Inflamm. Bowel Dis. 2012, 18, 63-73. [CrossRef]

68. Dubinsky, M.C.; Yang, H.; Hassard, P.V.; Seidman, E.G.; Kam, L.Y.; Abreu, M.T.; Targan, S.R.; Vasiliauskas, E.A. 6-MP metabolite profiles provide a biochemical explanation for 6-MP resistance in patients with inflammatory bowel disease. Gastroenterology 2002, 122, 904-915. [CrossRef] [PubMed]

69. Dubinsky, M.C.; Vasiliauskas, E.A.; Singh, H.; Abreu, M.T.; Papadakis, K.A.; Tran, T.; Martin, P.; Vierling, J.M.; Geller, S.A.; Targan, S.R.; et al. 6-thioguanine can cause serious liver injury in inflammatory bowel disease patients. Gastroenterology 2003, 125, 298-303. [CrossRef]

70. Fei, X.; Shu, Q.; Zhu, H.; Hua, B.; Wang, S.; Guo, L.; Fang, Y.; Ge, W. NUDT15 R139C Variants Increase the Risk of Azathioprine-Induced Leukopenia in Chinese Autoimmune Patients. Front. Pharmacol. 2018, 9, 460. [CrossRef] [PubMed]

71. Feng, R.; Guo, J.; Zhang, S.H.; Qiu, Y.; Chen, B.L.; He, Y.; Zeng, Z.R.; Ben-Horin, S.; Chen, M.H.; Mao, R. Low 6-thioguanine nucleotide level: Effective in maintaining remission in Chinese patients with Crohn's disease. J. Gastroenterol. Hepatol. 2019, 34, 679-685. [CrossRef]

72. Ferucci, E.D.; Hurlburt, K.J.; Mayo, M.J.; Livingston, S.; Deubner, H.; Gove, J.; Plotnik, J.; McMahon, B.J. Azathioprine metabolite measurements are not useful in following treatment of autoimmune hepatitis in Alaska Native and other non-Caucasian people. Can. J. Gastroenterol. 2011, 25, 21-27. [CrossRef]

73. Ganping, Z.; Zuofu, C.; Shedian, Z.; Zhichun, F.; Feng, X. A Simplistic Individualization Method for 6-Mercaptopurine in Acute Lymphoblastic Leukemia Children. Int. J. Pharmacol. 2008, 4, 64-66. [CrossRef] 
74. Gardiner, S.J.; Gearry, R.B.; Begg, E.J.; Zhang, M.; Barclay, M.L. Thiopurine dose in intermediate and normal metabolizers of thiopurine methyltransferase may differ three-fold. Clin. Gastroenterol. Hepatol. 2008, 6, 654-660. [CrossRef]

75. Gupta, P.; Gokhale, R.; Kirschner, B.S. 6-mercaptopurine metabolite levels in children with inflammatory bowel disease. J. Pediatr. Gastroenterol. Nutr. 2001, 33, 450-454. [CrossRef]

76. Halonen, P.; Mattila, J.; Makipernaa, A.; Ruuska, T.; Schmiegelow, K. Erythrocyte concentrations of metabolites or cumulative doses of 6-mercaptopurine and methotrexate do not predict liver changes in children treated for acute lymphoblastic leukemia. Pediatr. Blood Cancer 2006, 46, 762-766. [CrossRef]

77. Hande, S.; Wilson-Rich, N.; Bousvaros, A.; Zholudev, A.; Maurer, R.; Banks, P.; Makrauer, F.; Reddy, S.; Burakoff, R.; Friedman, S. 5-aminosalicylate therapy is associated with higher 6-thioguanine levels in adults and children with inflammatory bowel disease in remission on 6-mercaptopurine or azathioprine. Inflamm. Bowel Dis. 2006, 12, 251-257. [CrossRef] [PubMed]

78. Heerasing, N.M.; Ng, J.F.; Dowling, D. Does lymphopenia or macrocytosis reflect 6-thioguanine levels in patients with inflammatory bowel disease treated with azathioprine or 6-mercaptopurine? Intern. Med. J. 2016, 46, 465-469. [CrossRef] [PubMed]

79. Hindorf, U.; Lindqvist, M.; Hildebrand, H.; Fagerberg, U.; Almer, S. Adverse events leading to modification of therapy in a large cohort of patients with inflammatory bowel disease. Aliment. Pharmacol. Ther. 2006, 24, 331-342. [CrossRef] [PubMed]

80. Hindorf, U.; Lindqvist, M.; Peterson, C.; Soderkvist, P.; Strom, M.; Hjortswang, H.; Pousette, A.; Almer, S. Pharmacogenetics during standardised initiation of thiopurine treatment in inflammatory bowel disease. Gut 2006, 55, 1423-1431. [CrossRef] [PubMed]

81. Innocenti, F.; Danesi, R.; Favre, C.; Nardi, M.; Menconi, M.C.; Di Paolo, A.; Bocci, G.; Fogli, S.; Barbara, C.; Barachini, S.; et al. Variable correlation between 6-mercaptopurine metabolites in erythrocytes and hematologic toxicity: Implications for drug monitoring in children with acute lymphoblastic leukemia. Ther. Drug Monit. 2000, 22, 375-382. [CrossRef] [PubMed]

82. Kopylov, U.; Amre, D.; Theoret, Y.; Deslandres, C.; Seidman, E.G. Thiopurine metabolite ratios for monitoring therapy in pediatric Crohn disease. J. Pediatr. Gastroenterol. Nutr. 2014, 59, 511-515. [CrossRef]

83. Lancaster, D.L.; Lennard, L.; Rowland, K.; Vora, A.J.; Lilleyman, J.S. Thioguanine versus mercaptopurine for therapy of childhood lymphoblastic leukaemia: A comparison of haematological toxicity and drug metabolite concentrations. Br. J. Haematol. 1998, 102, 439-443. [CrossRef]

84. Lee, M.N.; Kang, B.; Choi, S.Y.; Kim, M.J.; Woo, S.Y.; Kim, J.W.; Choe, Y.H.; Lee, S.Y. Relationship between azathioprine dosage, 6-thioguanine nucleotide levels, and therapeutic response in pediatric patients with IBD treated with azathioprine. Inflamm. Bowel Dis. 2015, 21, 1054-1062. [CrossRef]

85. Lee, J.H.; Kim, T.J.; Kim, E.R.; Hong, S.N.; Chang, D.K.; Choi, L.H.; Woo, H.I.; Lee, S.Y.; Kim, Y.H. Measurements of 6-thioguanine nucleotide levels with TPMT and NUDT15 genotyping in patients with Crohn's disease. PLoS ONE 2017, 12, e0188925. [CrossRef]

86. Lennard, L.; Rees, C.A.; Lilleyman, J.S.; Maddocks, J.L. Childhood leukaemia: A relationship between intracellular 6-mercaptopurine metabolites and neutropenia. Br. J. Clin. Pharmacol. 1983, 16, 359-363. [CrossRef]

87. Lennard, L.; Brown, C.B.; Fox, M.; Maddocks, J.L. Azathioprine metabolism in kidney transplant recipients. Br. J. Clin. Pharmacol. 1984, 18, 693-700. [CrossRef] [PubMed]

88. Lennard, L.; Lilleyman, J.S.; Van Loon, J.; Weinshilboum, R.M. Genetic variation in response to 6-mercaptopurine for childhood acute lymphoblastic leukaemia. Lancet 1990, 336, 225-229. [CrossRef]

89. Lennard, L.; Richards, S.; Cartwright, C.S.; Mitchell, C.; Lilleyman, J.S.; Vora, A. The thiopurine methyltransferase genetic polymorphism is associated with thioguanine-related veno-occlusive disease of the liver in children with acute lymphoblastic leukemia. Clin. Pharmacol. Ther. 2006, 80, 375-383. [CrossRef]

90. Lilleyman, J.S.; Lennard, L.; Rees, C.A.; Morgan, G.; Maddocks, J.L. Childhood lymphoblastic leukaemia: Sex difference in 6-mercaptopurine utilization. Br. J. Cancer 1984, 49, 703-707. [CrossRef] [PubMed]

91. Liu, Q.; Wang, Y.; Mei, Q.; Han, W.; Hu, J.; Hu, N. Measurement of red blood cell 6-thioguanine nucleotide is beneficial in azathioprine maintenance therapy of Chinese Crohn's disease patients. Scand. J. Gastroenterol. 2016, 51, 1093-1099. [CrossRef] 
92. Melaouhia, S.; Fekih, M.; Ferchichi, H.; Bouissorra, H.; Ben Mustapha, N.; Boubaker, J.; Filali, A.; Lakhal, M.; Klouz, A. Is there any interest to dose the azathioprine's metabolites during inflammatory bowel diseases? Therapie 2013, 68, 313-320. [CrossRef] [PubMed]

93. Nguyen, T.M.; Le Gall, C.; Lachaux, A.; Boulieu, R. High thiopurine metabolite concentrations associated with lymphopenia in inflammatory bowel disease (IBD) pediatric patients receiving aminosalicylates combined with azathioprine. Int. J. Clin. Pharmacol. Ther. 2010, 48, 275-281. [CrossRef]

94. Nguyen, T.M.; Daubard, M.; Le Gall, C.; Larger, M.; Lachaux, A.; Boulieu, R. Monitoring of azathioprine metabolites in pediatric patients with autoimmune hepatitis. Ther. Drug Monit. 2010, 32, 433-437. [CrossRef]

95. Nygaard, U.; Toft, N.; Schmiegelow, K. Methylated metabolites of 6-mercaptopurine are associated with hepatotoxicity. Clin. Pharmacol. Ther. 2004, 75, 274-281. [CrossRef]

96. Odahara, S.; Uchiyama, K.; Kubota, T.; Ito, Z.; Takami, S.; Kobayashi, H.; Saito, K.; Koido, S.; Ohkusa, T. A Prospective Study Evaluating Metabolic Capacity of Thiopurine and Associated Adverse Reactions in Japanese Patients with Inflammatory Bowel Disease (IBD). PLoS ONE 2015, 10, e0137798. [CrossRef]

97. Ohtsuka, Y.; Arai, K.; Aoyagi, Y.; Fujii, T.; Yamakawa, Y.; Ohtani, K.; Ikuse, T.; Baba, Y.; Inage, E.; Kudo, T.; et al. Monitoring 6-thioguanine nucleotide concentrations in Japanese children and adolescents with inflammatory bowel disease. J. Gastroenterol. Hepatol. 2010, 25, 1626-1630. [CrossRef] [PubMed]

98. Ooi, C.Y.; Bohane, T.D.; Lee, D.; Naidoo, D.; Day, A.S. Thiopurine metabolite monitoring in paediatric inflammatory bowel disease. Aliment. Pharmacol. Ther. 2007, 25, 941-947. [CrossRef] [PubMed]

99. Pranzatelli, M.R.; Tate, E.D.; Allison, T.J. 6-Mercaptopurine modifies cerebrospinal fluid T cell abnormalities in paediatric opsoclonus-myoclonus as steroid sparer. Clin. Exp. Immunol. 2017, 190, 217-225. [CrossRef] [PubMed]

100. Rae, W.; Burke, G.; Pinto, A. A study of the utility of azathioprine metabolite testing in myasthenia gravis. J. Neuroimmunol. 2016, 293, 82-85. [CrossRef] [PubMed]

101. Schmiegelow, K.; Bruunshuus, I. 6-Thioguanine nucleotide accumulation in red blood cells during maintenance chemotherapy for childhood acute lymphoblastic leukemia, and its relation to leukopenia. Cancer Chemother. Pharmacol. 1990, 26, 288-292. [CrossRef]

102. Shaye, O.A.; Yadegari, M.; Abreu, M.T.; Poordad, F.; Simon, K.; Martin, P.; Papadakis, K.A.; Ippoliti, A.; Vasiliauskas, E.; Tran, T.T. Hepatotoxicity of 6-mercaptopurine (6-MP) and Azathioprine (AZA) in adult IBD patients. Am. J. Gastroenterol. 2007, 102, 2488-2494. [CrossRef] [PubMed]

103. Wojtuszkiewicz, A.; Barcelos, A.; Dubbelman, B.; De Abreu, R.; Brouwer, C.; Bokkerink, J.P.; de Haas, V.; de Groot-Kruseman, H.; Jansen, G.; Kaspers, G.L.; et al. Assessment of mercaptopurine (6MP) metabolites and 6MP metabolic key-enzymes in childhood acute lymphoblastic leukemia. Nucleosides Nucleotides Nucleic Acids 2014, 33, 422-433. [CrossRef]

104. Wong, D.R.; Coenen, M.J.; Derijks, L.J.; Vermeulen, S.H.; van Marrewijk, C.J.; Klungel, O.H.; Scheffer, H.; Franke, B.; Guchelaar, H.J.; de Jong, D.J.; et al. Early prediction of thiopurine-induced hepatotoxicity in inflammatory bowel disease. Aliment. Pharmacol. Ther. 2017, 45, 391-402. [CrossRef]

105. Wong, D.R.; Coenen, M.J.; Vermeulen, S.H.; Derijks, L.J.; van Marrewijk, C.J.; Klungel, O.H.; Scheffer, H.; Franke, B.; Guchelaar, H.J.; de Jong, D.J.; et al. Early Assessment of Thiopurine Metabolites Identifies Patients at Risk of Thiopurine-induced Leukopenia in Inflammatory Bowel Disease. J. Crohns Colitis 2017, 11, 175-184. [CrossRef]

106. Wright, S.; Sanders, D.S.; Lobo, A.J.; Lennard, L. Clinical significance of azathioprine active metabolite concentrations in inflammatory bowel disease. Gut 2004, 53, 1123-1128. [CrossRef]

107. Yarur, A.J.; Gondal, B.; Hirsch, A.; Christensen, B.; Cohen, R.D.; Rubin, D.T. Higher Thioguanine Nucleotide Metabolite Levels Are Associated with Better Long-Term Outcomes in Patients with Inflammatory Bowel Diseases. J. Clin. Gastroenterol. 2018, 52, 537-544. [CrossRef]

108. Zochowska, D.; Zegarska, J.; Hryniewiecka, E.; Samborowska, E.; Jazwiec, R.; Tszyrsznic, W.; Borowiec, A.; Dadlez, M.; Paczek, L. Determination of Concentrations of Azathioprine Metabolites 6-Thioguanine and 6-Methylmercaptopurine in Whole Blood With the Use of Liquid Chromatography Combined With Mass Spectrometry. Transplant. Proc. 2016, 48, 1836-1839. [CrossRef] [PubMed]

109. Van Asseldonk, D.P.; Jharap, B.; Verheij, J.; den Hartog, G.; Westerveld, D.B.; Becx, M.C.; Russel, M.G.; Engels, L.G.; de Jong, D.J.; Witte, B.I.; et al. The Prevalence of Nodular Regenerative Hyperplasia in Inflammatory Bowel Disease Patients Treated with Thioguanine Is Not Associated with Clinically Significant Liver Disease. Inflamm. Bowel Dis. 2016, 22, 2112-2120. [CrossRef] 
110. Kennedy, N.A.; Heap, G.A.; Green, H.D.; Hamilton, B.; Bewshea, C.; Walker, G.J.; Thomas, A.; Nice, R.; Perry, M.H.; Bouri, S.; et al. Predictors of anti-TNF treatment failure in anti-TNF-naive patients with active luminal Crohn's disease: A prospective, multicentre, cohort study. Lancet Gastroenterol. 2019, 4, 341-353. [CrossRef]

111. Roblin, X.; Williet, N.; Boschetti, G.; Phelip, J.M.; Del Tedesco, E.; Berger, A.E.; Vedrines, P.; Duru, G.; Peyrin-Biroulet, L.; Nancey, S.; et al. Addition of azathioprine to the switch of anti-TNF in patients with IBD in clinical relapse with undetectable anti-TNF trough levels and antidrug antibodies: A prospective randomised trial. Gut 2020, 69, 1206-1212. [CrossRef]

112. Yarur, A.J.; Kubiliun, M.J.; Czul, F.; Sussman, D.A.; Quintero, M.A.; Jain, A.; Drake, K.A.; Hauenstein, S.I.; Lockton, S.; Deshpande, A.R.; et al. Concentrations of 6-thioguanine nucleotide correlate with trough levels of infliximab in patients with inflammatory bowel disease on combination therapy. Clin. Gastroenterol. Hepatol. 2015, 13, 1118-1124. [CrossRef] [PubMed]

113. Roblin, X.; Boschetti, G.; Williet, N.; Nancey, S.; Marotte, H.; Berger, A.; Phelip, J.M.; Peyrin-Biroulet, L.; Colombel, J.F.; Del Tedesco, E.; et al. Azathioprine dose reduction in inflammatory bowel disease patients on combination therapy: An open-label, prospective and randomised clinical trial. Aliment. Pharmacol. Ther. 2017, 46, 142-149. [CrossRef] [PubMed]

114. De Boer, N.K.; de Graaf, P.; Wilhelm, A.J.; Mulder, C.J.; van Bodegraven, A.A. On the limitation of 6-tioguaninenucleotide monitoring during tioguanine treatment. Aliment. Pharmacol. Ther. 2005, 22, 447-451. [CrossRef] [PubMed]

115. Duley, J.A.; Florin, T.H. Thiopurine therapies: Problems, complexities, and progress with monitoring thioguanine nucleotides. Ther. Drug Monit. 2005, 27, 647-654. [CrossRef]

116. Dervieux, T.; Blanco, J.G.; Krynetski, E.Y.; Vanin, E.F.; Roussel, M.F.; Relling, M.V. Differing contribution of thiopurine methyltransferase to mercaptopurine versus thioguanine effects in human leukemic cells. Cancer Res. 2001, 61, 5810-5816.

117. Lim, S.Z.; Chua, E.W. Revisiting the Role of Thiopurines in Inflammatory Bowel Disease through Pharmacogenomics and Use of Novel Methods for Therapeutic Drug Monitoring. Front. Pharmacol. 2018, 9, 1107. [CrossRef]

118. Fraser, A.G.; Jewell, D.P. Side effects of azathioprine treatment given for inflammatory bowel disease-a 30 year audit. Gastroenterology 2000, 118, A787. [CrossRef]

119. McGovern, D.P.B.; Travis, S.P.L.; Duley, J.; Shobowale-Bakre, E.M.; Dalton, H.R. Azathioprine intolerance in patients with IBD may be imidazole-related and is independent of TPMT activity. Gastroenterology 2002, 122, 838-839. [CrossRef] [PubMed]

120. Domenech, E.; Nos, P.; Papo, M.; Lopez-San Roman, A.; Garcia-Planella, E.; Gassull, M.A. 6-mercaptopurine in patients with inflammatory bowel disease and previous digestive intolerance of azathioprine. Scand. J. Gastroenterol. 2005, 40, 52-55. [CrossRef] [PubMed]

121. Shi, H.Y.; Chan, F.K.L.; Leung, W.K.; Li, M.K.K.; Leung, C.M.; Sze, S.F.; Ching, J.Y.L.; Lo, F.H.; Tsang, S.W.C.; Shan, E.H.S.; et al. Low-dose azathioprine is effective in maintaining remission in steroid-dependent ulcerative colitis: Results from a territory-wide Chinese population-based IBD registry. Therap. Adv. Gastroenterol. 2016, 9, 449-456. [CrossRef] [PubMed]

(C) 2020 by the authors. Licensee MDPI, Basel, Switzerland. This article is an open access article distributed under the terms and conditions of the Creative Commons Attribution (CC BY) license (http://creativecommons.org/licenses/by/4.0/). 